\title{
Toward Covalent Organic Framework Metastructures
}

Song Wang, ${ }^{1 \dagger}$ Yuhao Yang, ${ }^{1,2 \dagger}$ Haoran Zhang, ${ }^{1}$ Ziyang Zhang, ${ }^{1}$ Chi Zhang, ${ }^{1}$ Xiaodong Huang, ${ }^{1}$ Daichi Kozawa, ${ }^{3}$ Pingwei Liu,,${ }^{1,2,}{ }^{*}$ Bo-Geng Li, ${ }^{1}$ and Wen-Jun Wang1,2

1 State Key Lab of Chemical Engineering, College of Chemical and Biological Engineering, Zhejiang University, Hangzhou, China, 310027

${ }^{2}$ Institute of Zhejiang University - Quzhou, 78 Jiuhua Boulevard North, Quzhou 324000, China

${ }^{3}$ Center for Advanced Photonics, RIKEN, 2-1 Hirosawa Wako, Saitama 351-0198, Japan

$\dagger$ These authors contribute equally

* Corresponding author: liupingwei@zju.edu.cn 


\section{Materials}

Mesitylene (98\%), benzaldehyde (98\%), aniline (99.5\%), scandium (III) trifluoromethanesulfonate $\left(\mathrm{Sc}(\mathrm{OTf})_{3}, 98 \%\right)$, tris(4-aminophenyl)-amine (TAPA 97\%), 1,3,5-tris(4-aminophenyl)-benzene (TAPB, 98\%), silver trifluoroacetate (98\%), hydrogen tetrachloroaurate(III) trihydrate $(98 \%)$, iodine $(99.8 \%)$ were purchased from J\&K Scientific Co.. 1,4-dioxane (99\%), methanol anhydrous (99.5\%), chloroform (99\%), hexane (97\%), sodium borohydride, were purchased from Sinopharm Chemical Reagent Co., Ltd. Tri(4-formlphenyl)-amine (TFPA 98\%), 1,3,5-tris(4-formlphenyl)benzene (TFPB, 98\%), 2,4,6-tris(4-aminophenyl)-1,3,5-triazine (TAPT 98\%), 2,4,6tris(4-formlphenyl)-1,3,5-triazine (TFPT 98\%), were purchased from ATK Chemical Company Limited. UV curable photopolymer NOA-61 was purchased from Norland Products Inc.. Trinitrophenol standard solution $(1 \mathrm{mg} / \mathrm{mL}$ in acetonitrile) was purchased from the resources platform of the national standard material. All reagents and solvents were used without further purification.

\section{Instrumentation}

Powder x-ray diffraction (PXRD). PXRD diffraction spectra were obtained with a PANalytical X-pert Powder diffractometer (Cu anode) using a Scan step of $0.0262606^{\circ}$ and step time of $97.983 \mathrm{~s}$.

Field emission scanning electron microscopy (FESEM). FESEM images were collected with a Hitachi SU-8010 field emission scanning electron microscope with accelerating voltage of $3 \mathrm{kV}$. All the samples were sprayed by Pt for $60 \mathrm{~s}$.

Transmission electron microscope (TEM). TEM images were obtained using a HT7700 transmission electron microscope from HITACHI company with an accelerating voltage of $100 \mathrm{kV}$. Energy Dispersive X-ray Spectroscopy (EDS) mapping was combined with a X-MAX ${ }^{\mathrm{n}} 65 \mathrm{~T}$ from Oxford Instruments with an accelerating voltage 
of $100 \mathrm{kV}$.

$\mathbf{N}_{2}$ absorption-desorption analysis. The absorption-desorption isotherms were collected by a AUTOSORB-IQ2-MP Surface Area and Porosimetry Analyzer. All the samples were kept at $120^{\circ} \mathrm{C}$ for $12 \mathrm{~h}$ for dewatering. $\mathrm{N}_{2}$ isotherms were measured at 77 K and analyzed by ASiQwin software. Pore width was calculated by QSDFT model.

Fourier transform infrared (FT-IR) spectroscopy. FT-IR spectra were measured with a Nicolet5700 FT-IR spectrometer.

Optical Microscope. The potical images were captured on digital optical microscope(Keyence VHX-7000, Japan).

Polarized optical microscopy. The polarized microscopy images were captured on depolarized transmission light microscope (DTLM) (CX40P, Sunny Optical Technology, China) equipped with a digital CCD camera (Oplenic, China) in transmission modes. The brightness of polarized microscopy images was measured by Image $\mathrm{J}$ software.

Micro-Area Refection and Transmission spectra. The micro-area refection and transmission spectra were measured with a full spectrum analyzing system in microregion (gora-Lite, ideaoptics, china).

Ink-Jet Printer. The ink-jet printer used is a SonoPlot Microplotter Proto, which is operated with the SonoGuide and SonoDraw software provided. The inkjet nozzle is composed of a glass capillary attached to an ultrasonicating piezoelectric device. 
Tubular Muffle Furnace. Samples were heated at a rate of $5^{\circ} \mathrm{C} \min ^{-1}$ up to $500^{\circ} \mathrm{C}$, kept at $500^{\circ} \mathrm{C}$ for $1 \mathrm{~h}$ and then heated at a rate of $5^{\circ} \mathrm{C} \min ^{-1}$ up to $900^{\circ} \mathrm{C}$, kept at $900^{\circ} \mathrm{C}$ for $8 \mathrm{~h}$. The products were cooled down slowly to room temperature. The atmosphere for the whole process is Ar.

\section{Experimental Methods}

\section{Synthesis of TAPA-TFPA COF flower}

Experiments were conducted at different temperatures with the same other conditions. Take the experiment at $25^{\circ} \mathrm{C}$ as example. $58.1 \mathrm{mg}$ of TAPA $(0.2 \mathrm{mmol})$ and $65.9 \mathrm{mg}$ of TFPA ( $0.2 \mathrm{mmol})$ were dissolved in $40 \mathrm{~mL}$ dioxane/mesitylene $(2 / 3, \mathrm{v} / \mathrm{v})$ assisted by sonication. $364.3 \mu \mathrm{L}$ of benzaldehyde $(3.60 \mathrm{mmol})$ and $326.4 \mu \mathrm{L}$ of aniline $(3.60 \mathrm{mmol})$ were added to the solution. $\mathrm{Sc}(\mathrm{OTf})_{3}(12 \mathrm{mg}, 16.4 \mu \mathrm{mol})$ in $0.6 \mathrm{~mL}$ of dioxane/mesitylene $(2 / 1, \mathrm{v} / \mathrm{v})$ was added to the mixture to start the reaction at $25^{\circ} \mathrm{C}$. After 3 days, the precipitate was collected by filtration and washed with dioxane/mesitylene $(2 / 3, v / v)$ and hexane. The product was dried in the fume hood overnight and in vacuum oven at $60^{\circ} \mathrm{C}$ for 1 days.

\section{Synthesis of TAPA-TFPA COF with different combinations of monofunctional competitors}

In the experiments, varied combinations of competitors (aniline and benzaldehyde) were employed. Take 18 equiv. aniline and 0 equiv. benzaldehyde as an example. 58.1 $\mathrm{mg}$ of TAPA $(0.2 \mathrm{mmol})$ and $65.9 \mathrm{mg}$ of TFPA $(0.2 \mathrm{mmol})$ were dissolved in $40 \mathrm{~mL}$ dioxane/mesitylene (2/3, v/v) assisted by sonication. $326.4 \mu \mathrm{L}$ of aniline $(3.60 \mathrm{mmol})$ were added to the solution. $\mathrm{Sc}(\mathrm{OTf})_{3}(12 \mathrm{mg}, 16.4 \mu \mathrm{mol})$ in $0.6 \mathrm{~mL}$ of dioxane/mesitylene $(2 / 1, \mathrm{v} / \mathrm{v})$ was added to the mixture to start the reaction at $25^{\circ} \mathrm{C}$. After 3 days, the precipitate was collected by filtration and washed with 
dioxane/mesitylene $(2 / 3, v / v)$ and hexane. The product was dried in the fume hood overnight and in vacuum oven at $60{ }^{\circ} \mathrm{C}$ for 1 days.

\section{Synthesis of TAPA-TFPA COF with different catalyst concentrations}

In the experiments, varied amounts of catalyst $\left(\mathrm{Sc}(\mathrm{OTf})_{3}\right)$ were employed. Take 0.3 $\mathrm{mg} / \mathrm{mL}$ concentration of $\mathrm{Sc}(\mathrm{OTf})_{3}$ as an example. $58.1 \mathrm{mg}$ of TAPA $(0.2 \mathrm{mmol})$ and $65.9 \mathrm{mg}$ of TFPA $(0.2 \mathrm{mmol})$ were dissolved in $40 \mathrm{~mL}$ dioxane/mesitylene $(2 / 3, \mathrm{v} / \mathrm{v})$ assisted by sonication. $326.4 \mu \mathrm{L}$ of aniline $(3.60 \mathrm{mmol})$ and $326.4 \mu \mathrm{L}$ of aniline $(3.60$ mmol) were added to the solution. $\mathrm{Sc}(\mathrm{OTf})_{3}(12 \mathrm{mg}, 16.4 \mu \mathrm{mol})$ in $0.6 \mathrm{~mL}$ of dioxane/mesitylene $(2 / 1, \mathrm{v} / \mathrm{v})$ was added to the mixture to start the reaction at $25^{\circ} \mathrm{C}$. After 3 days, the precipitate was collected by filtration and washed with dioxane/mesitylene $(2 / 3, v / v)$ and hexane. The product was dried in the fume hood overnight and in vacuum oven at $60^{\circ} \mathrm{C}$ for 1 days.

\section{Synthesis of TAPA-TFPA COF with different solvent ratios}

In the experiments, different solvent ratios for dioxane and mesitylene was employed. Take dioxane/mesitylene $(1 / 1, \mathrm{v} / \mathrm{v})$ as an example. $58.1 \mathrm{mg}$ of TAPA $(0.2 \mathrm{mmol})$ and $65.9 \mathrm{mg}$ of TFPA $(0.2 \mathrm{mmol})$ were dissolved in $40 \mathrm{~mL}$ dioxane/mesitylene $(1 / 1, \mathrm{v} / \mathrm{v})$ assisted by sonication. $326.4 \mu \mathrm{L}$ of aniline $(3.60 \mathrm{mmol})$ and $326.4 \mu \mathrm{L}$ of aniline $(3.60$ mmol) were added to the solution. $\mathrm{Sc}(\mathrm{OTf})_{3}(12 \mathrm{mg}, 16.4 \mu \mathrm{mol})$ in $0.6 \mathrm{~mL}$ of dioxane/mesitylene $(2 / 1, \mathrm{v} / \mathrm{v})$ was added to the mixture to start the reaction at $25{ }^{\circ} \mathrm{C}$. After 3 days, the precipitate was collected by filtration and washed with dioxane/mesitylene $(1 / 1, \mathrm{v} / \mathrm{v})$ and hexane. The product was dried in the fume hood overnight and in vacuum oven at $60{ }^{\circ} \mathrm{C}$ for 1 days. 


\section{Synthesis of TAPA-TFPB COF, TAPA-TFPT COF, TAPB-TFPA COF, TAPB- TFPB COF, TAPB-TFPT COF, TAPT-TFPA COF, TAPT-TFPB COF and TAPT-TFPT COF}

TAPA-TFPB COF, TAPA-TFPT COF, TAPB-TFPA COF, TAPB-TFPB COF, TAPBTFPT COF, TAPT-TFPA COF, TAPT-TFPB COF and TAPT-TFPT COF were synthesised following the similar procedure with different amino monomers (TAPA, TAPB and TAPT) and aldehyde monomers (TFPA, TFPB and TFPT) in the same reaction conditions. Take TAPT-TFPT COF as example, $70.9 \mathrm{mg}$ of TAPT ( $0.2 \mathrm{mmol})$ and $78.7 \mathrm{mg}$ of TFPT $(0.2 \mathrm{mmol})$ were dispersed in $40 \mathrm{~mL}$ dioxane/mesitylene $(2 / 3$, $\mathrm{v} / \mathrm{v})$ assisted by sonication. $364.3 \mu \mathrm{L}$ of benzaldehyde $(3.60 \mathrm{mmol})$ and $326.4 \mu \mathrm{L}$ of aniline $(3.60 \mathrm{mmol})$ were added to the solution. $\mathrm{Sc}(\mathrm{OTf})_{3}(12 \mathrm{mg}, 16.4 \mu \mathrm{mol})$ in $0.6 \mathrm{~mL}$ of dioxane $\quad /$ mesitylene $(2 / 1, \mathrm{v} / \mathrm{v})$ was added to the mixture to start the reaction at $25^{\circ} \mathrm{C}$. After 3 days, the precipitate was collected by filtration and washed with dioxane/mesitylene $(2 / 3, v / v)$ and hexane. The product was dried in the fume hood overnight and in vacuum oven at $60^{\circ} \mathrm{C}$ for 1 days.

\section{Evolution of TAPA-TFPA COF flower}

$58.1 \mathrm{mg}$ of TAPA $(0.2 \mathrm{mmol})$ and $65.9 \mathrm{mg}$ of TFPA $(0.2 \mathrm{mmol})$ were dissolved in 40 $\mathrm{mL}$ dioxane/mesitylene $(2 / 3, \mathrm{v} / \mathrm{v})$ assisted by sonication. $364.3 \mu \mathrm{L}$ of benzaldehyde (3.60 mmol) and $326.4 \mu \mathrm{L}$ of aniline $(3.60 \mathrm{mmol})$ were added to the solution. $\mathrm{Sc}(\mathrm{OTf})_{3}$ (12 mg, $16.4 \mu \mathrm{mol})$ in $0.6 \mathrm{~mL}$ of dioxane/mesitylene $(2 / 1, \mathrm{v} / \mathrm{v})$ was added to the mixture to start the reaction at $25{ }^{\circ} \mathrm{C}$. The precipitates after different reaction times were collected by filtration and washed with dioxane/mesitylene $(2 / 3, v / v)$ and hexane. The product was dried in the fume hood overnight and in vacuum oven at $60{ }^{\circ} \mathrm{C}$ for 1 days.

\section{Synthesis of Au/Ag@TAPA-TFPA COF flower}


4mg TAPA-TFPA COF synthesized at $25^{\circ} \mathrm{C}$ was dispersed in $5 \mathrm{~mL}$ methanol assisted by sonication. Hydrogen tetrachloroaurate(III) trihydrate $(1.0 \mathrm{mg}) / \mathrm{silver}$ trifluoroacetate $(0.6 \mathrm{mg})$ in $0.1 \mathrm{ml}$ of methanol was added to the dispersion. Sodium borohydride $(1 \mathrm{mg})$ in $0.1 \mathrm{ml}$ of methanol was added slowly to the mixture to recover gold/sliver. The precipitate at given time was collected by filtration and washed with methanol. The product was dried in the fume hood overnight and in vacuum oven at 60 ${ }^{\circ} \mathrm{C}$ for 1 days.

\section{Trinitrophenol sensing of TAPA-TFPA COF petal}

COF petals were dispersed on a glass slide and measured by polarized optical microscopy at brightest angle. One drop of trinitrophenol solution in chloroform with different concentration was dropped on the glass slide and dried in hood. Then the COF petals on glass slide was measured by polarized optical microscopy at brightest angle again with the same parameters. The brightness was quantized by Image $\mathbf{J}$ software. 
A

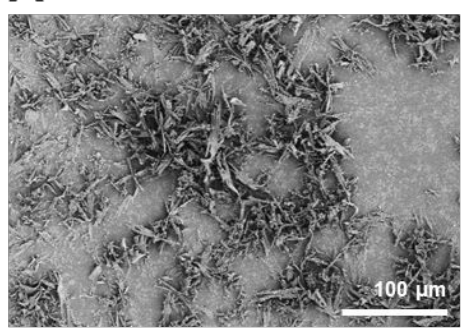

B

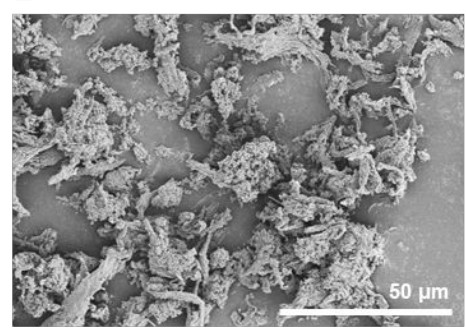

C

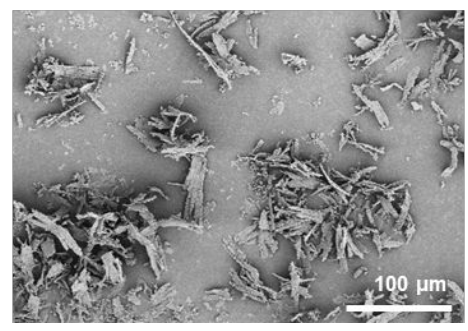

No aniline or benzaldehyde
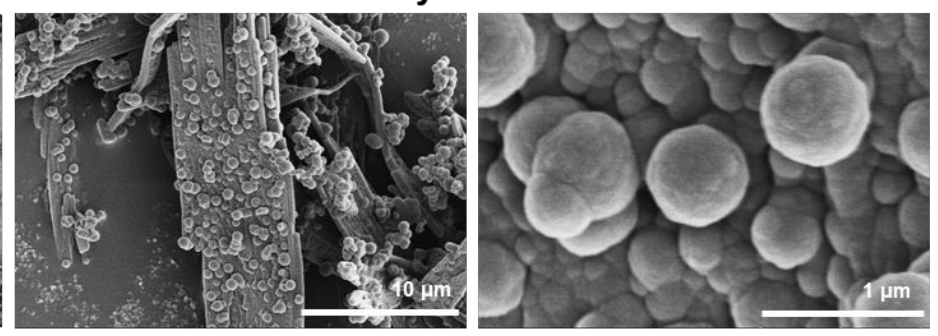

\section{Only benzaldehyde}

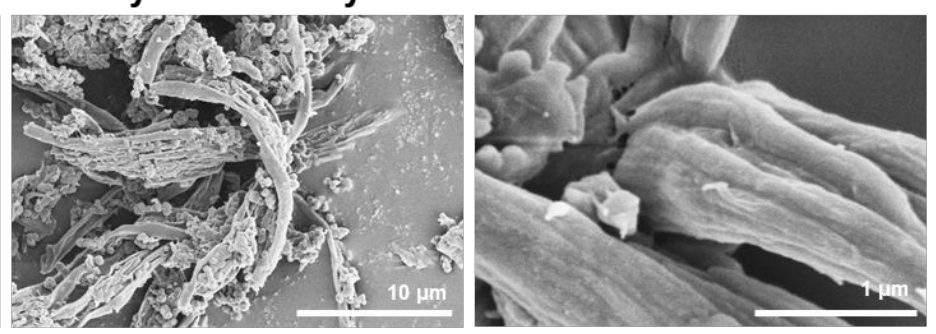

\section{Only aniline}
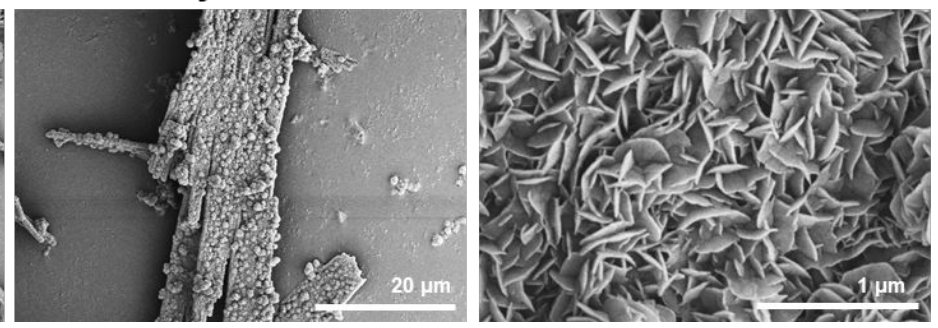

Supplementary Figure 1. FESEM images of the TAPA-TFPA COF synthesized without benzaldehyde or aniline (A), with only benzaldehyde (B) and only aniline (C). All the other conditions are the same with that of TAPA-TFPA COF flower. Products synthesized with only benzaldehyde or without adding any competitors are random aggregates containing no nanoflakes, and those synthesized with only aniline present disordered nanoflakes 

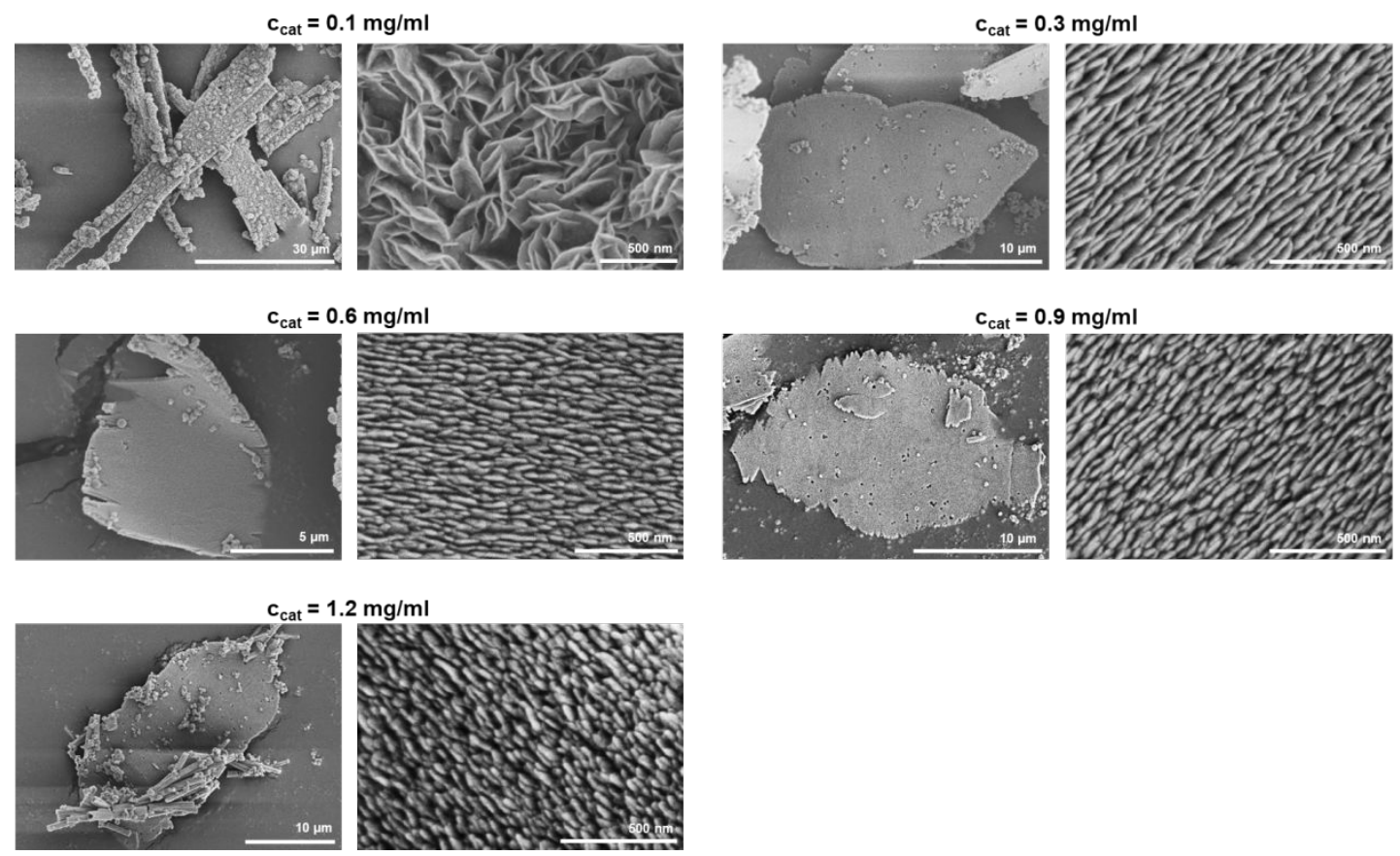

Supplementary Figure 2. FESEM images of the TAPA-TFPA COF synthesized with different catalyst concentration $\left(\mathrm{c}_{\mathrm{cat}}\right)$. Other conditions are the same: monomer concentration is $5 \mathrm{mM}$ in Dioxane/mesitylene (2/3, v/v) with $90 \mathrm{mM}$ benzaldehyde and aniline. Products with disordered nanoflakes are also obtained when the concentration of catalyst is $0.1 \mathrm{mg} / \mathrm{mL}$, however, sizes of nanoflakes will decrease when the concentration goes higher than $0.3 \mathrm{mg} / \mathrm{mL}$ 


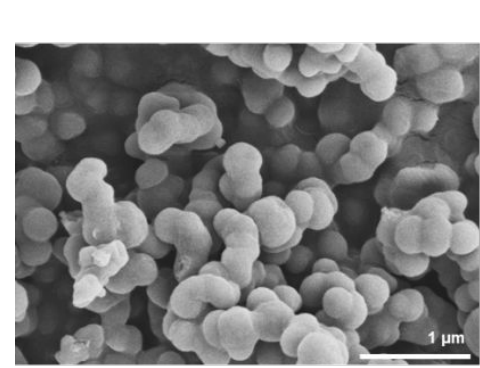

Dioxane/Mesitylene (2/1, v/v)

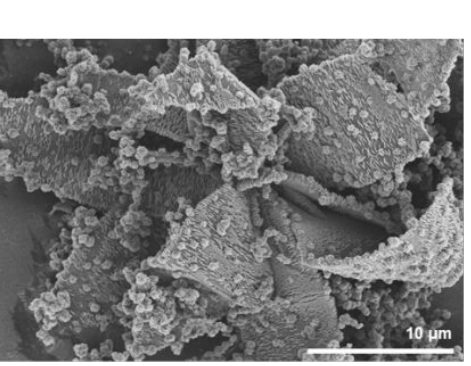

Dioxane/Mesitylene $(1 / 1, v / v)$
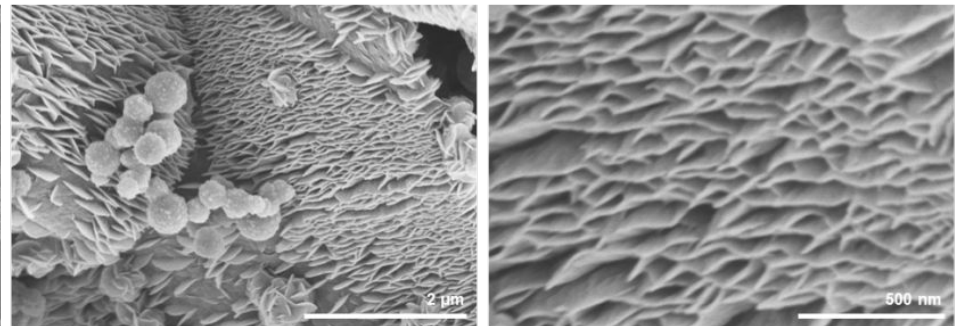

Dioxane/Mesitylene (1/2, v/v)
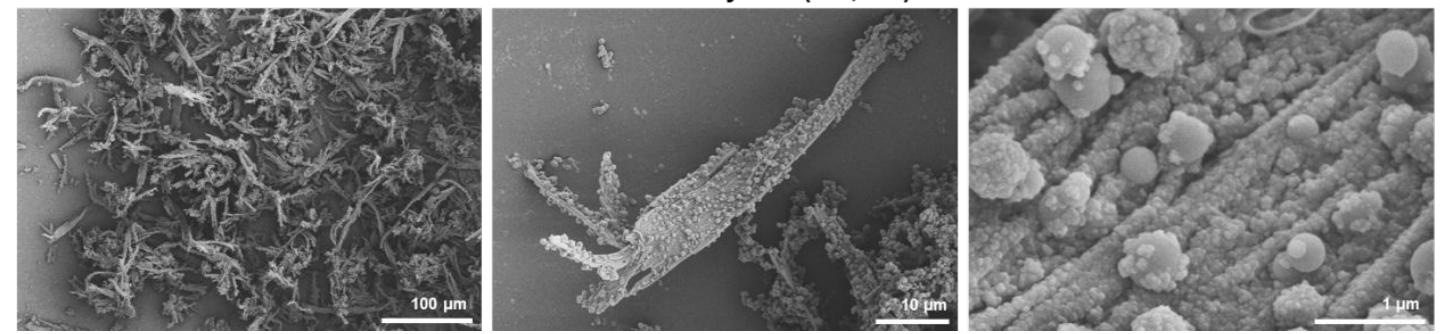

Supplementary Figure 3. FESEM images of the TAPA-TFPA COF synthesized with different solvent ratio. Other conditions are the same: monomer concentration is $5 \mathrm{mM}$ benzaldehyde and aniline concentration is $90 \mathrm{mM}$. catalyst concentration is $0.3 \mathrm{mg} / \mathrm{mL}$. At dioxane/mesitylene ratio of $2 / 3(\mathrm{v} / \mathrm{v})$, the product presents dense nanoflakes, and sparse or no nanoflakes will be obtained at other ratio, which might be attributed to the mismatch of surface tension. 
A
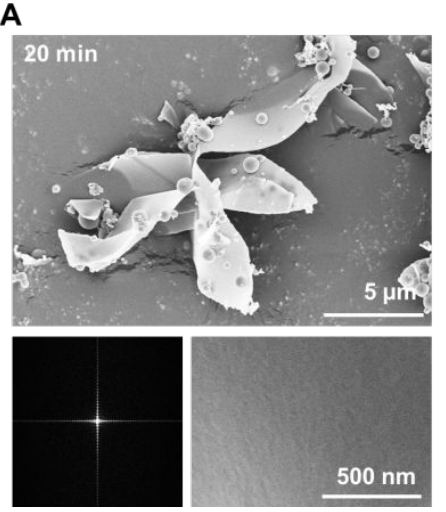

B
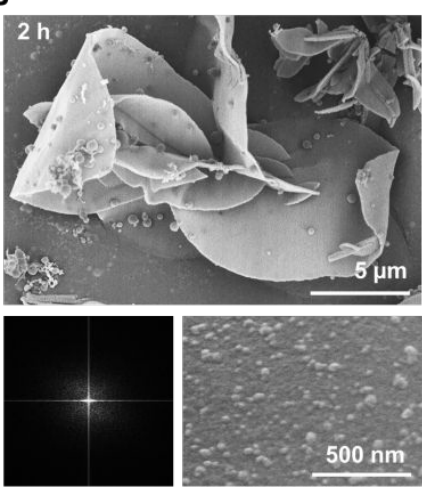

C
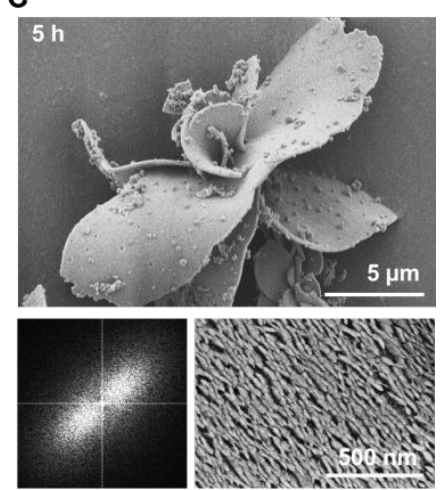

Supplementary Figure 4. FESEM images and FFT of nanoflakes evolution on TAPATFPA COF flower at different times.

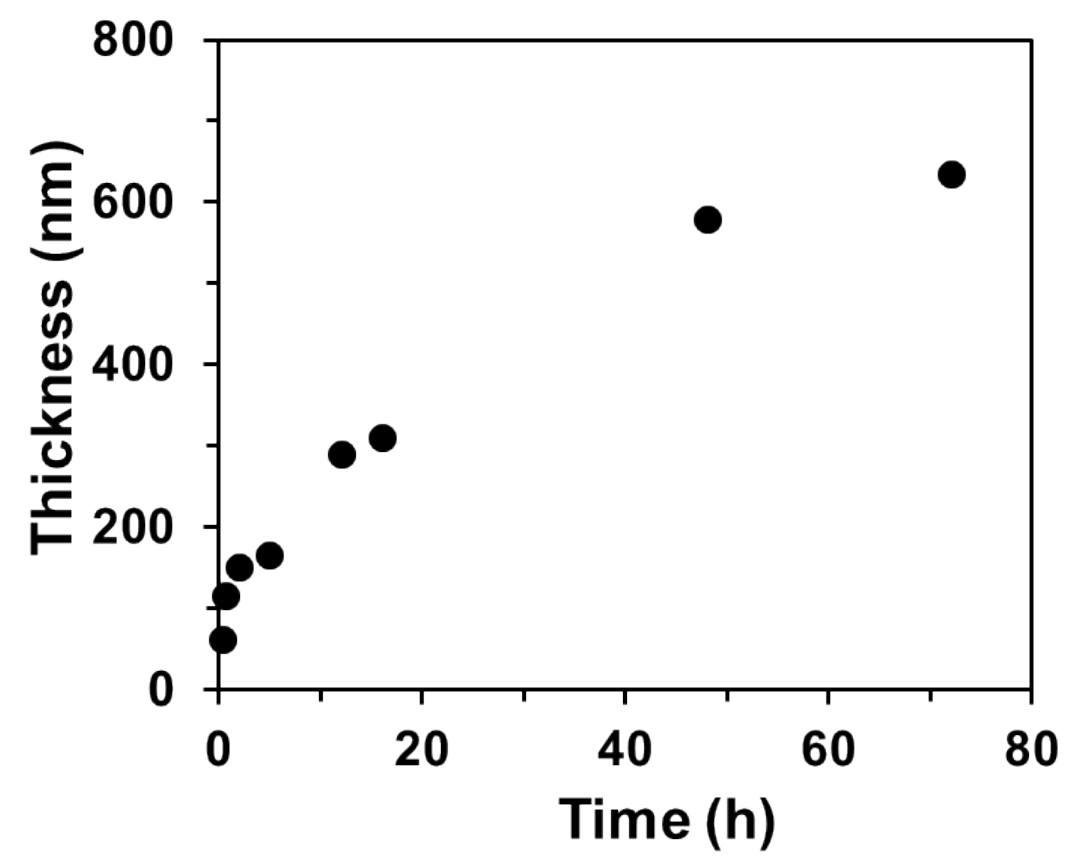

Supplementary Figure 5. FESEM images and FFT of nanoflakes evolution on TAPATFPA COF "petals" at different times. 

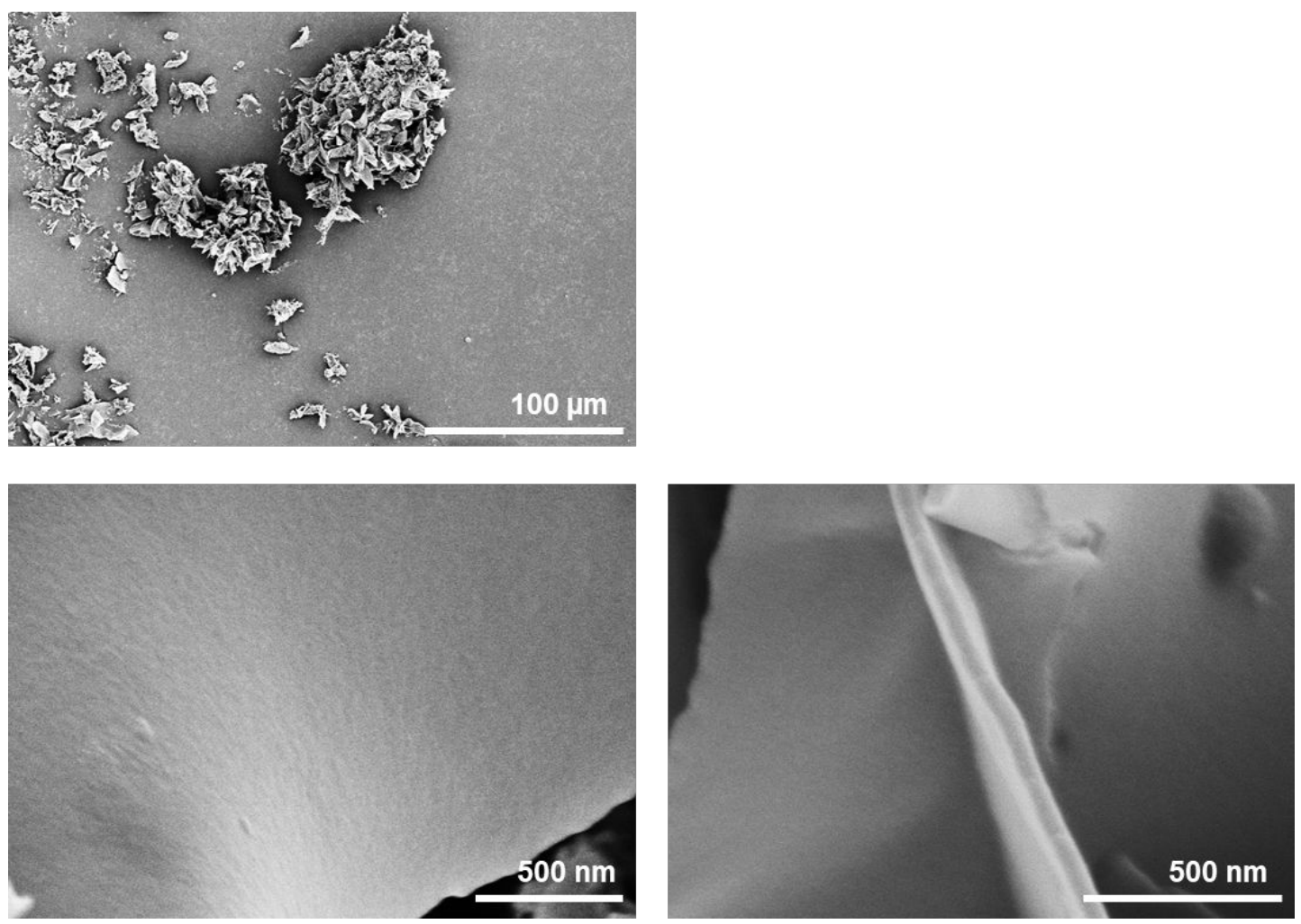

Supplementary Figure 6. FESEM images of TAPA-TFPA COF flower synthesized at room temperature for $20 \mathrm{~min}$.
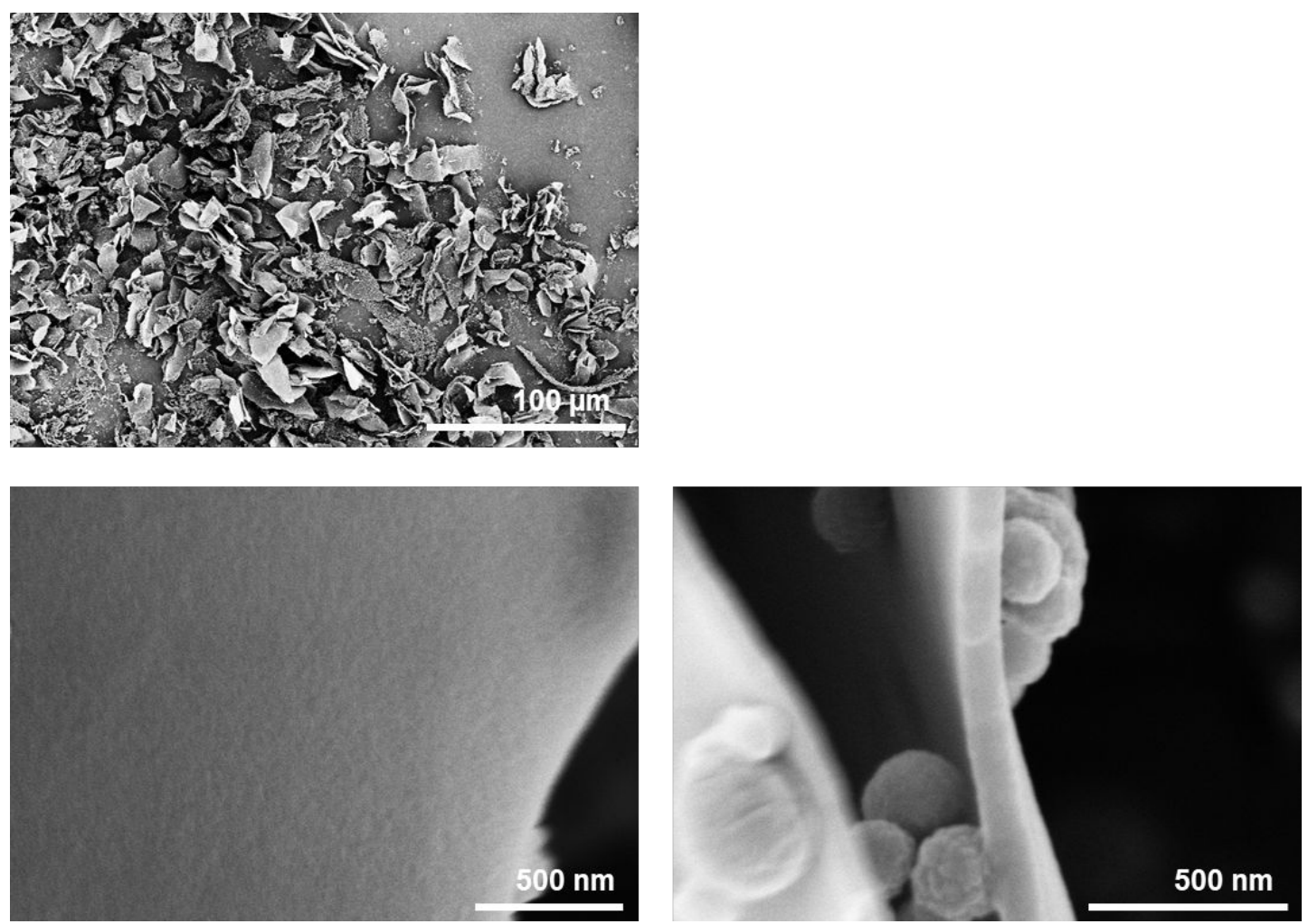

Supplementary Figure 7. FESEM images of TAPA-TFPA COF flower synthesized at room temperature for $40 \mathrm{~min}$. 

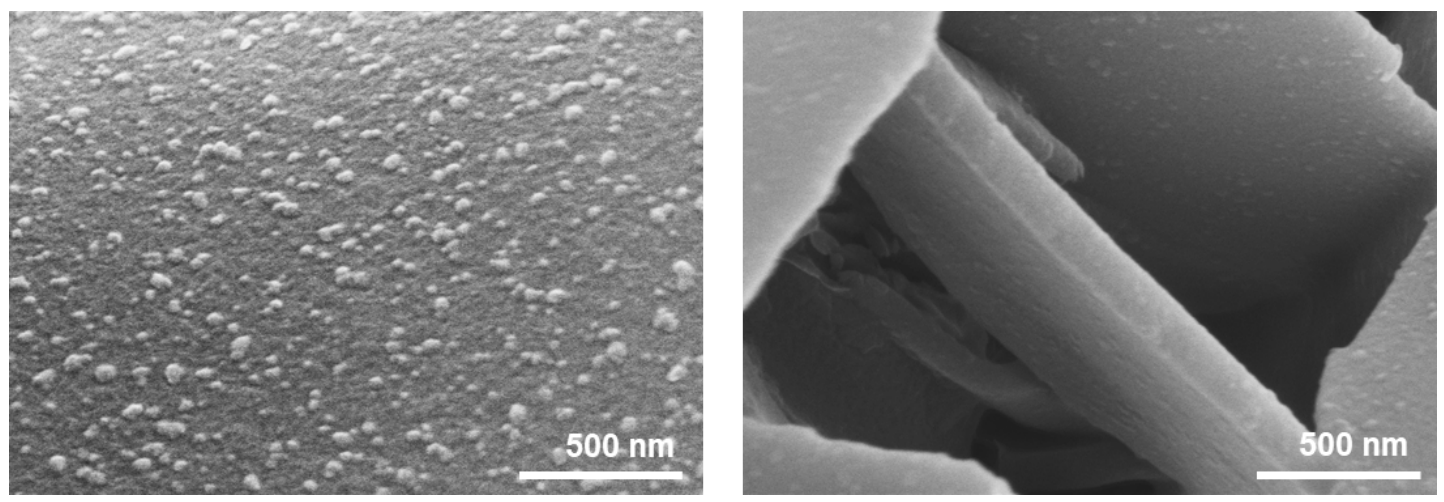

Supplementary Figure 8. FESEM images of TAPA-TFPA COF flower synthesized at room temperature for $2 \mathrm{~h}$.
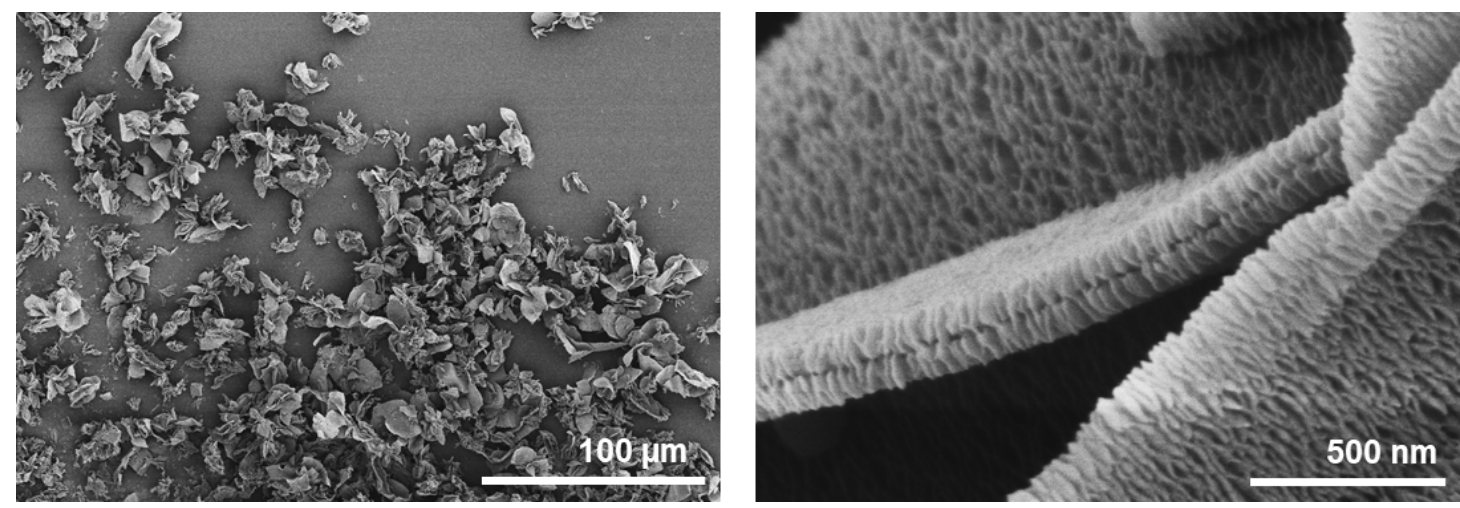

Supplementary Figure 9. FESEM images of TAPA-TFPA COF flower synthesized at room temperature for $5 \mathrm{~h}$. 

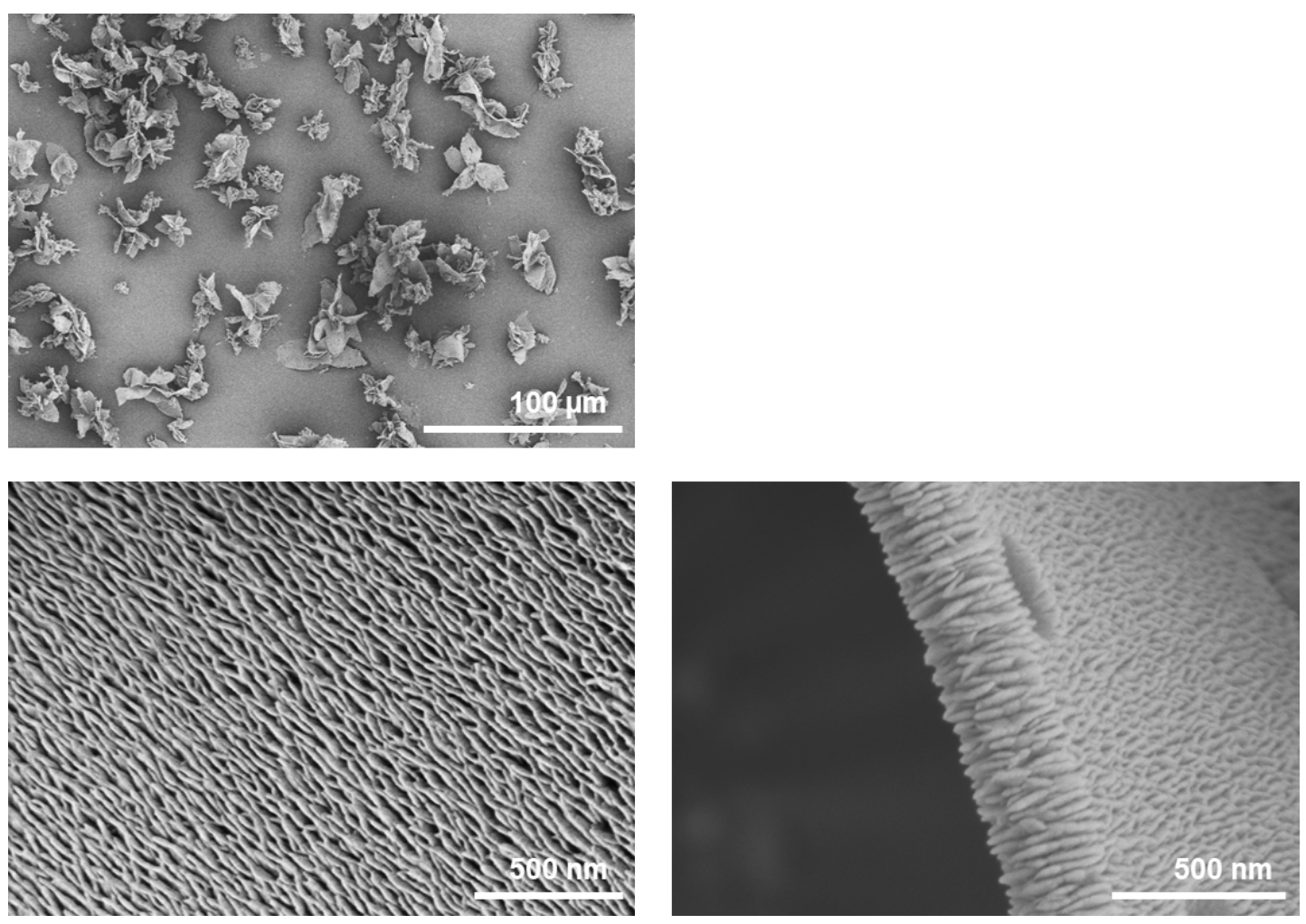

Supplementary Figure 10. FESEM images of TAPA-TFPA COF flower synthesized at room temperature for $12 \mathrm{~h}$.
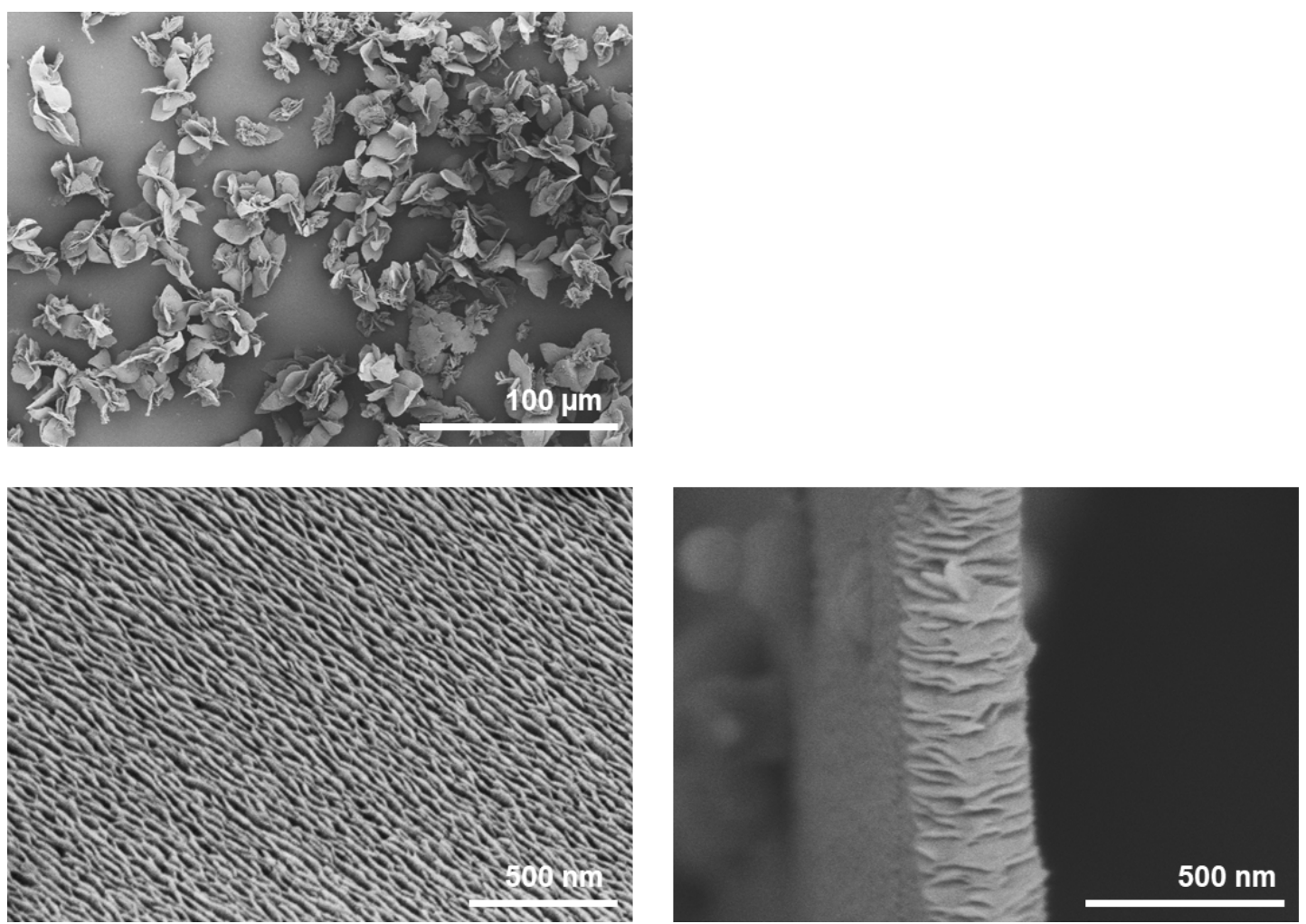

Supplementary Figure 11. FESEM images of TAPA-TFPA COF flower synthesized at room temperature for $16 \mathrm{~h}$. 

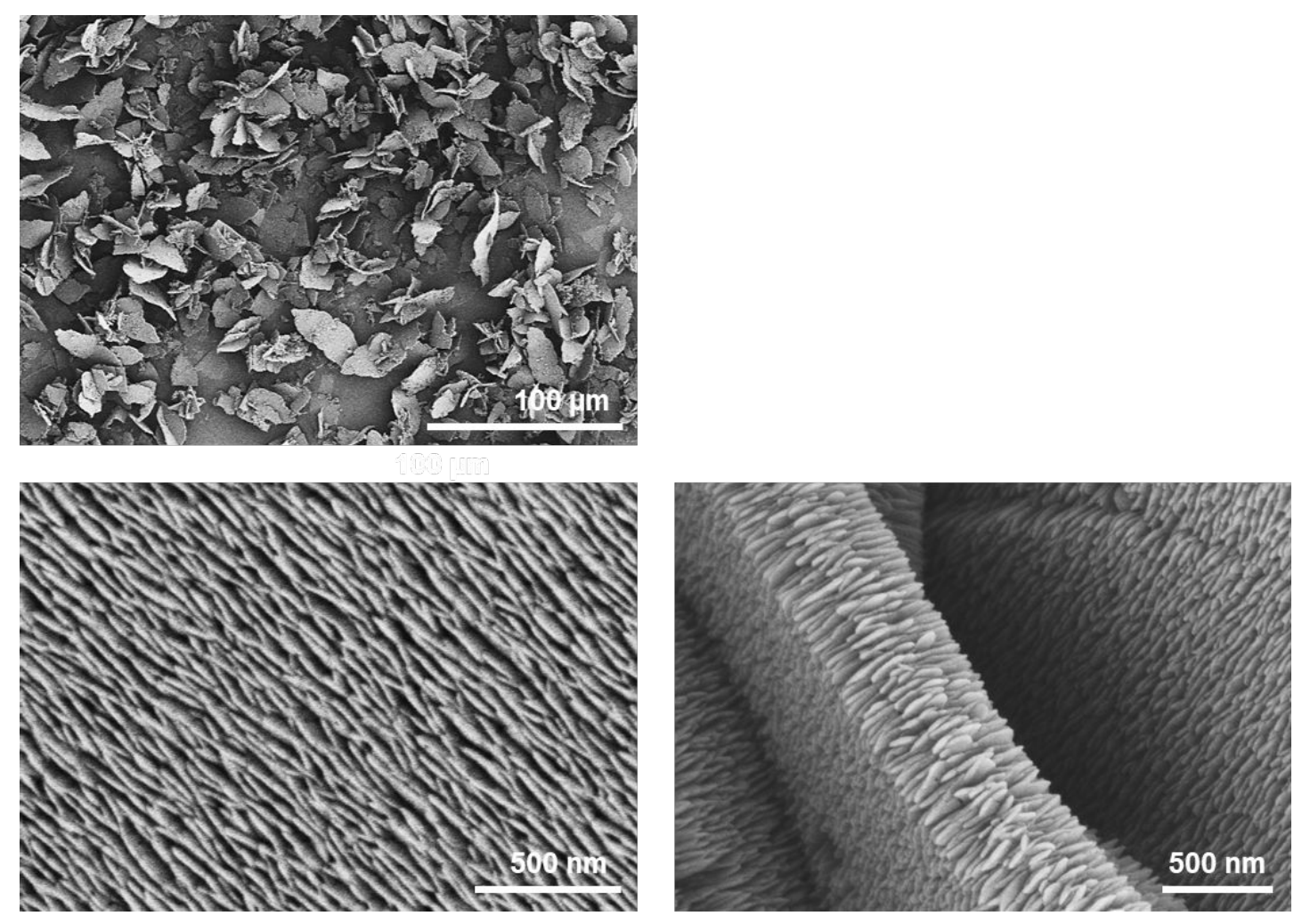

Supplementary Figure 12. FESEM images of TAPA-TFPA COF flower synthesized at room temperature for $48 \mathrm{~h}$.
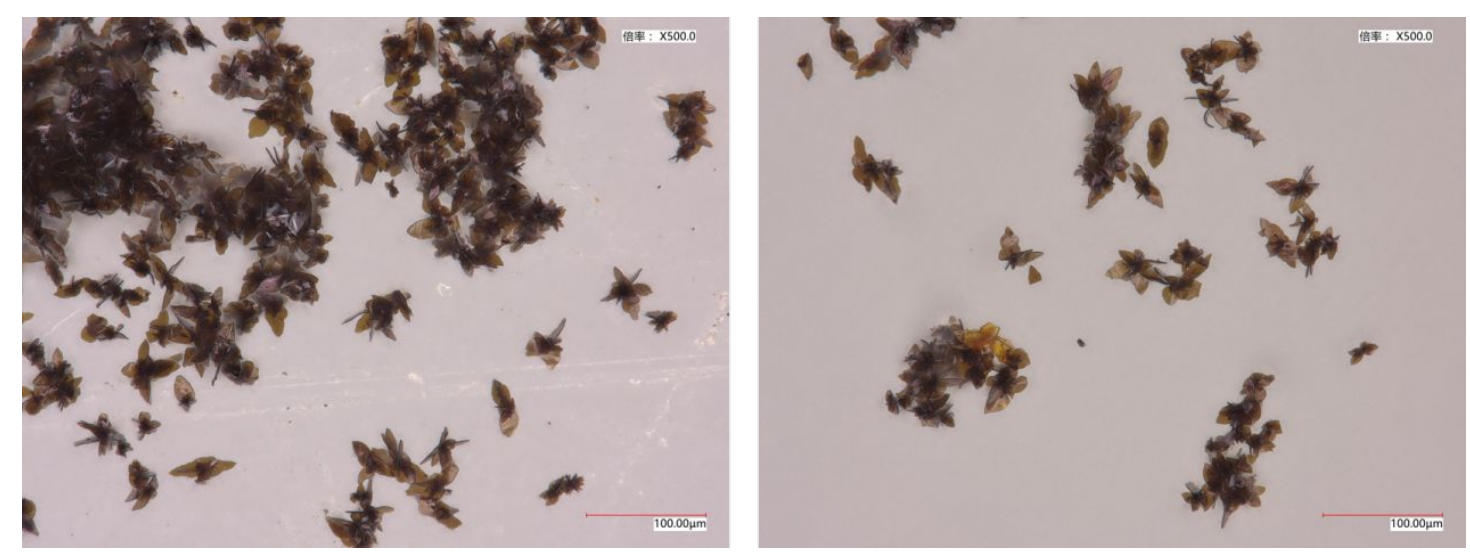

Supplementary Figure 13. Optical microscope images of the TAPA-TFPA COF flower in different scales. Conditions: monomer concentration is $5 \mathrm{mM}$, benzaldehyde and aniline concentration is $90 \mathrm{mM}$. catalyst concentration is $0.3 \mathrm{mg} / \mathrm{mL}$, solvent is dioxane/mesitylene mixture $(2 / 3, \mathrm{v} / \mathrm{v})$. 

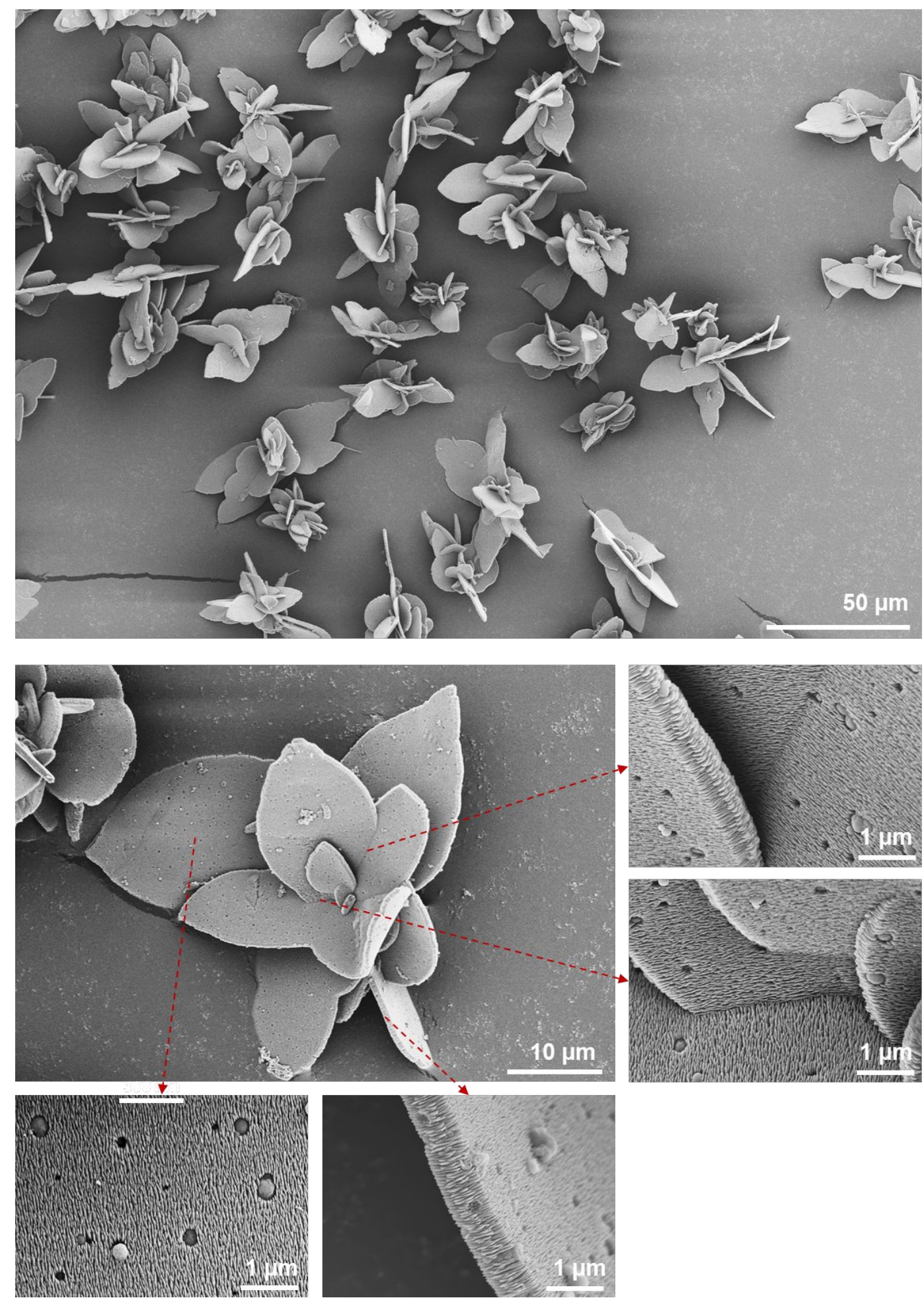

Supplementary Figure 14. FESEM images of the TAPA-TFPA COF flower synthesized at room temperature in different scales. 


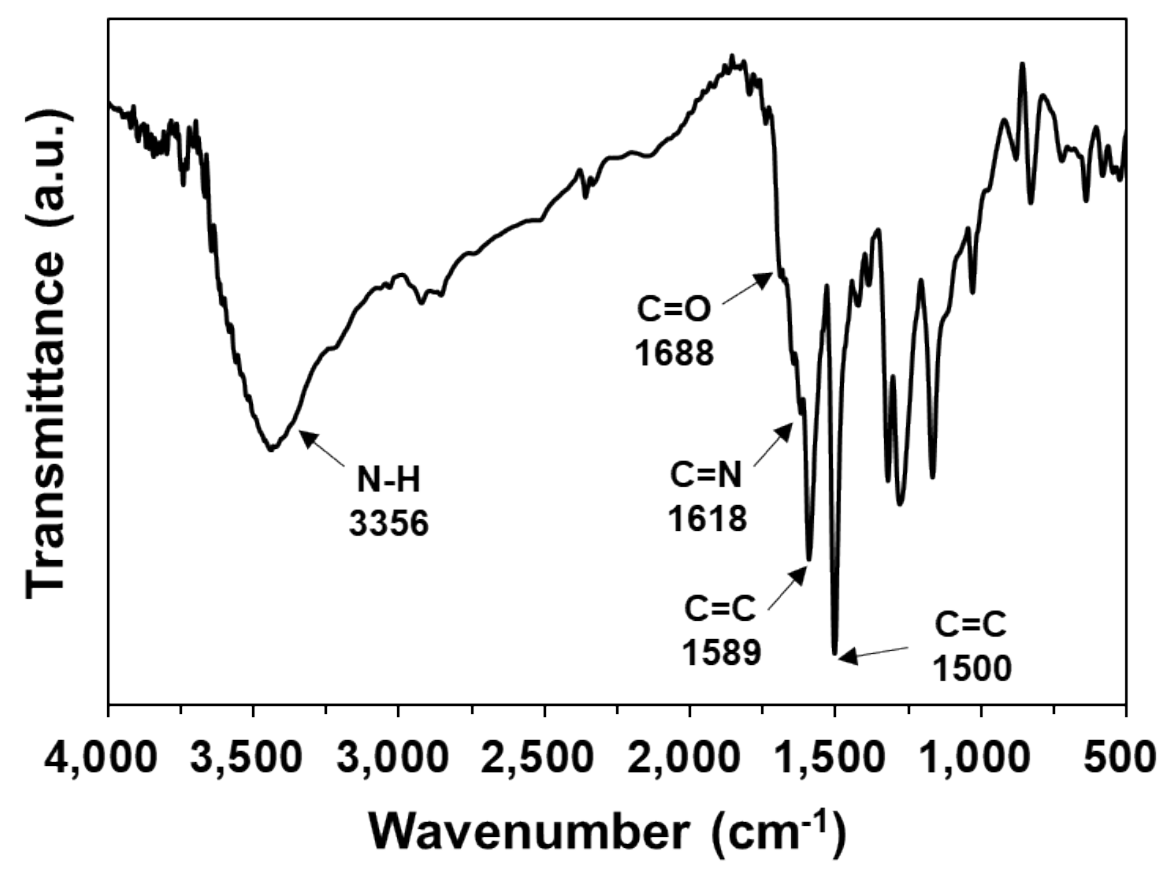

Supplementary Figure 15. FT-IR spectrum of TAPA-TFPA COF flower synthesized at room temperautre.

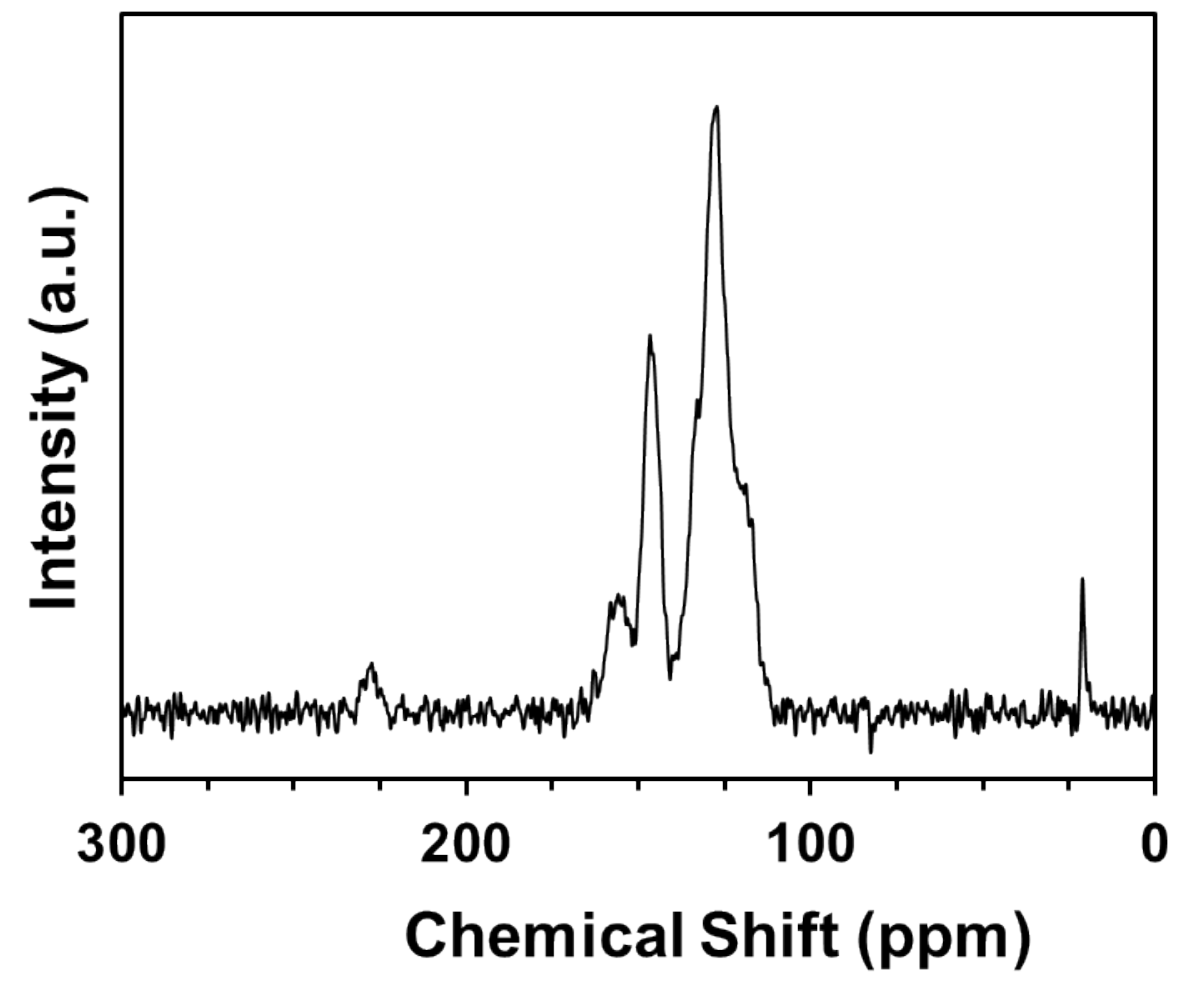

Supplementary Figure 16. Solid state ${ }^{13} \mathrm{C}$ CP MAS NMR spectrum of TAPA-TFPA COF flower synthesized at room temperautre. 


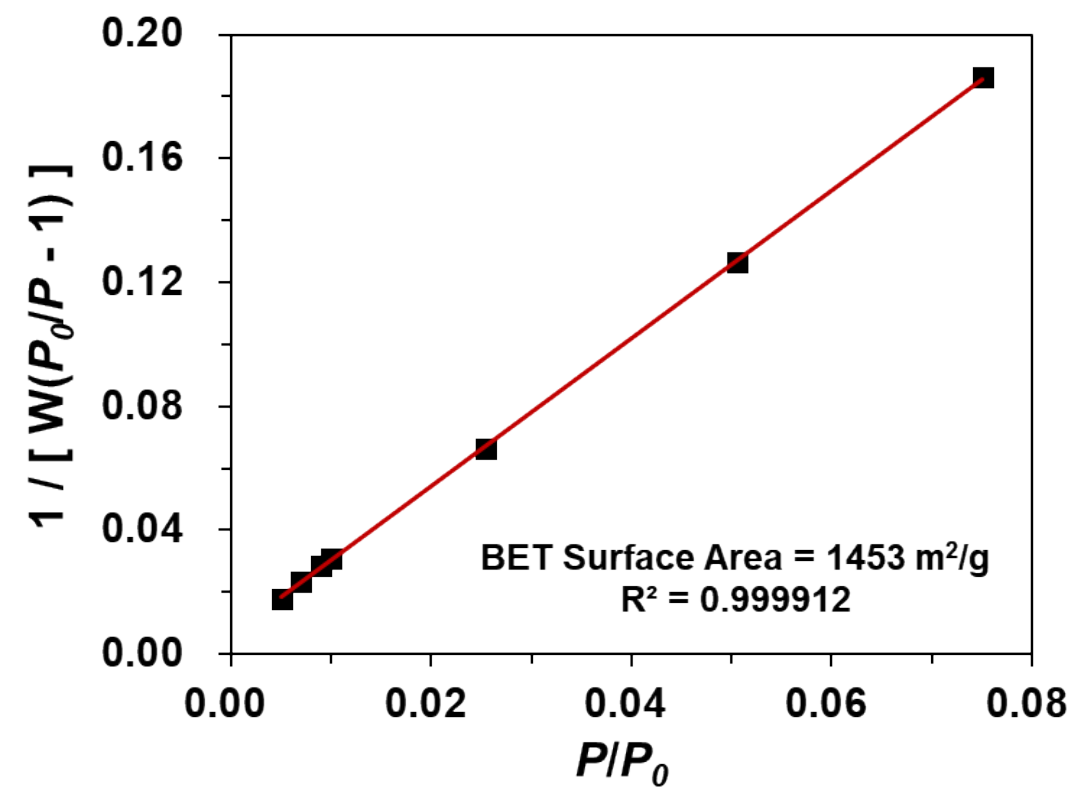

Supplementary Figure 17. BET plot for TAPA-TFPA COF flower synthesized at room temperature calculated from nitrogen adsorption data.

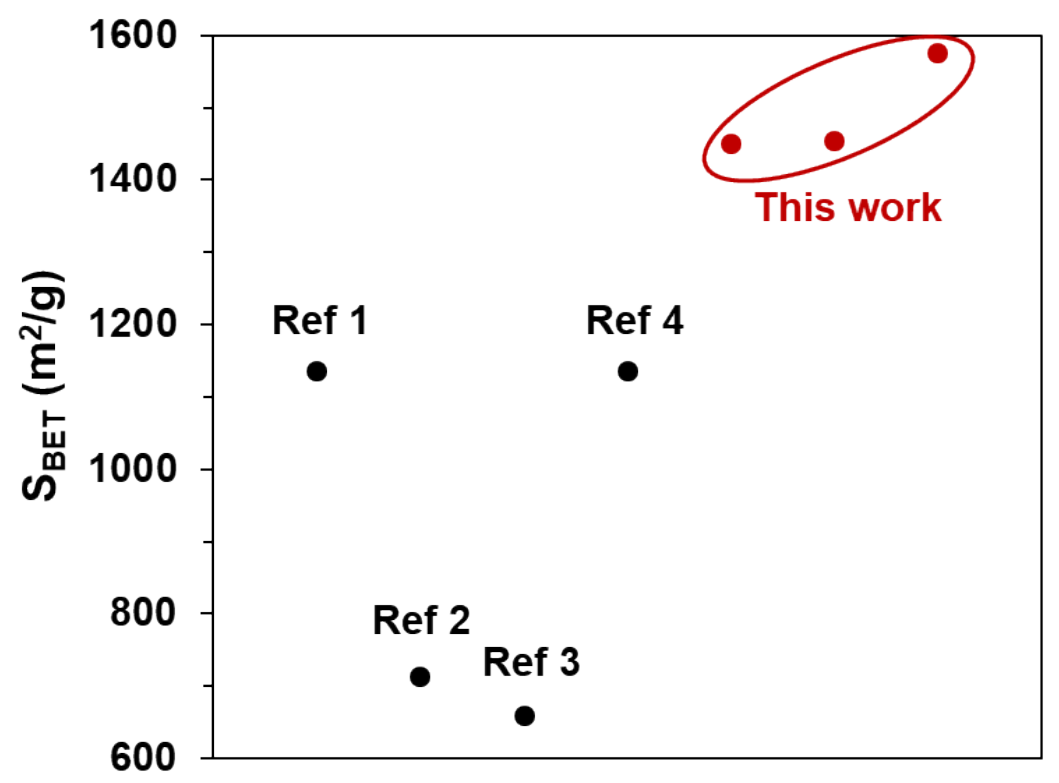

Supplementary Figure 18. Master plot of BET surface area $\left(\mathrm{S}_{\mathrm{BET}}\right)$ of the TAPATFPA COFs. ${ }^{1,2}$ 


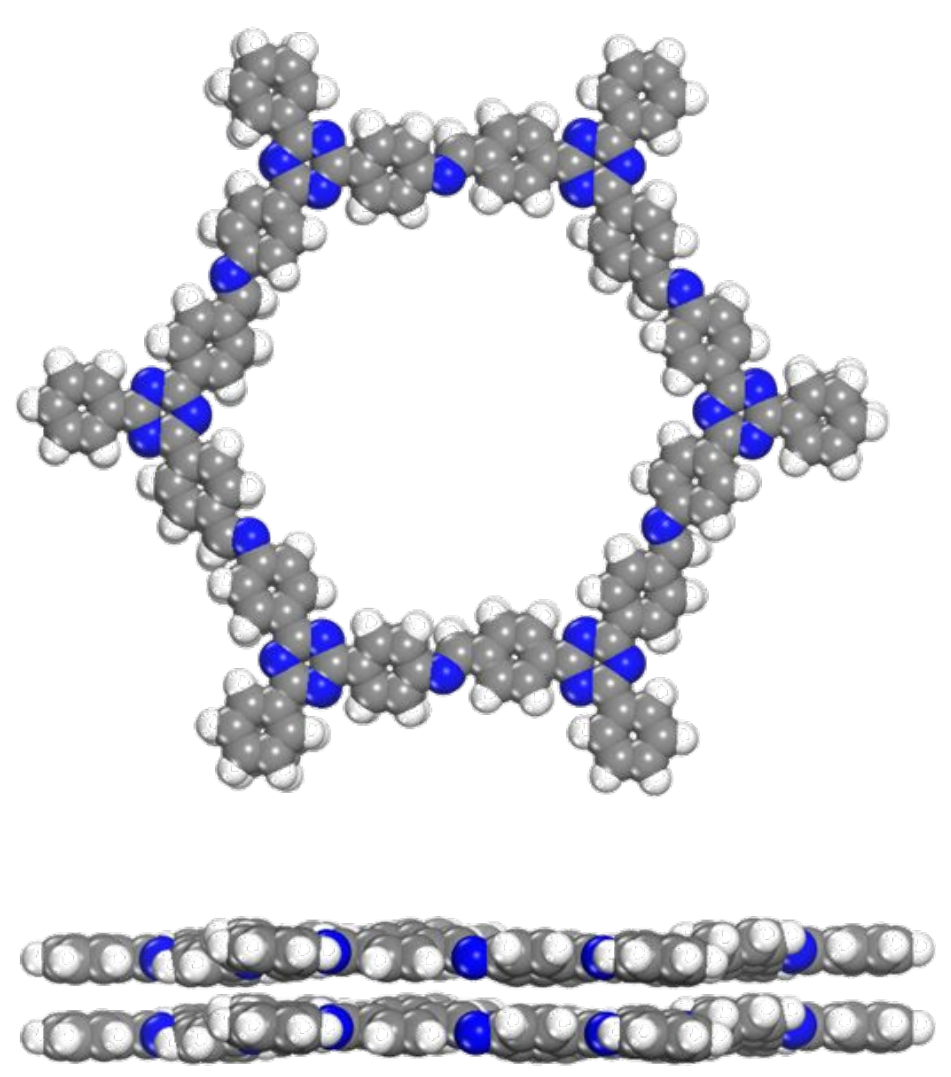

\section{Stacking energy per hexagon bilayer is $536 \mathrm{~kJ} / \mathrm{mol}$}

Supplementary Figure 19. Energy minimized hexagonally stacked bilayer (top and side views) of TAPT-TFPT COF based on DFT (Density Functional Theory). Energies of each layer and the bilayer were calculated in a development version of ADF, using the PBE functional including Grimme dispersion correction (i.e. PBE-D3) and DZ basis set. Stacking energies are thus calculated as E(bilayer) $-[\mathrm{E}($ top $)+\mathrm{E}$ (bottom) . 

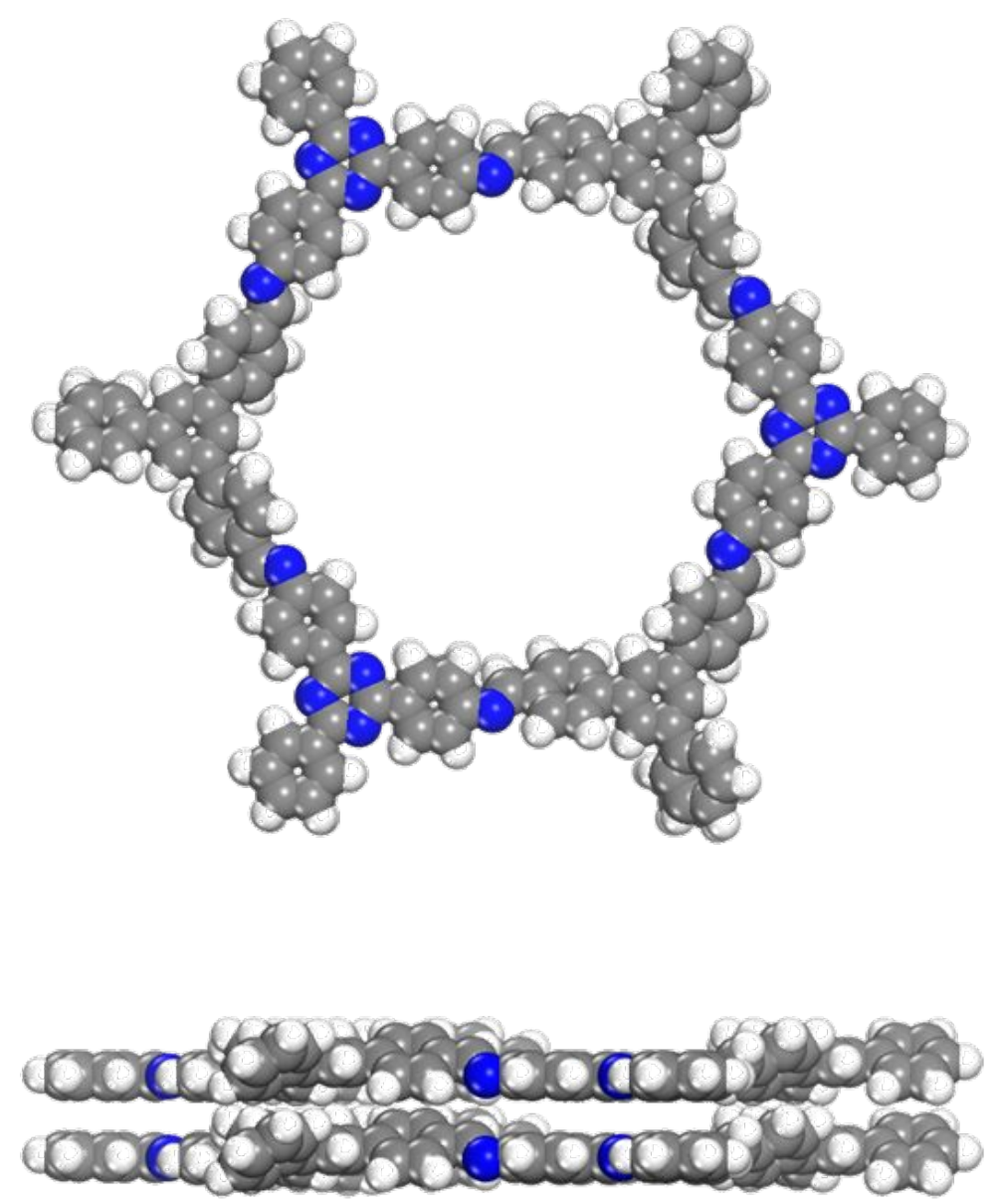

\section{Stacking energy per hexagon bilayer is $523 \mathrm{~kJ} / \mathrm{mol}$}

Supplementary Figure 20. Energy minimized hexagonally stacked bilayer (top and side views) of TAPT-TFPB COF based on DFT (Density Functional Theory). 

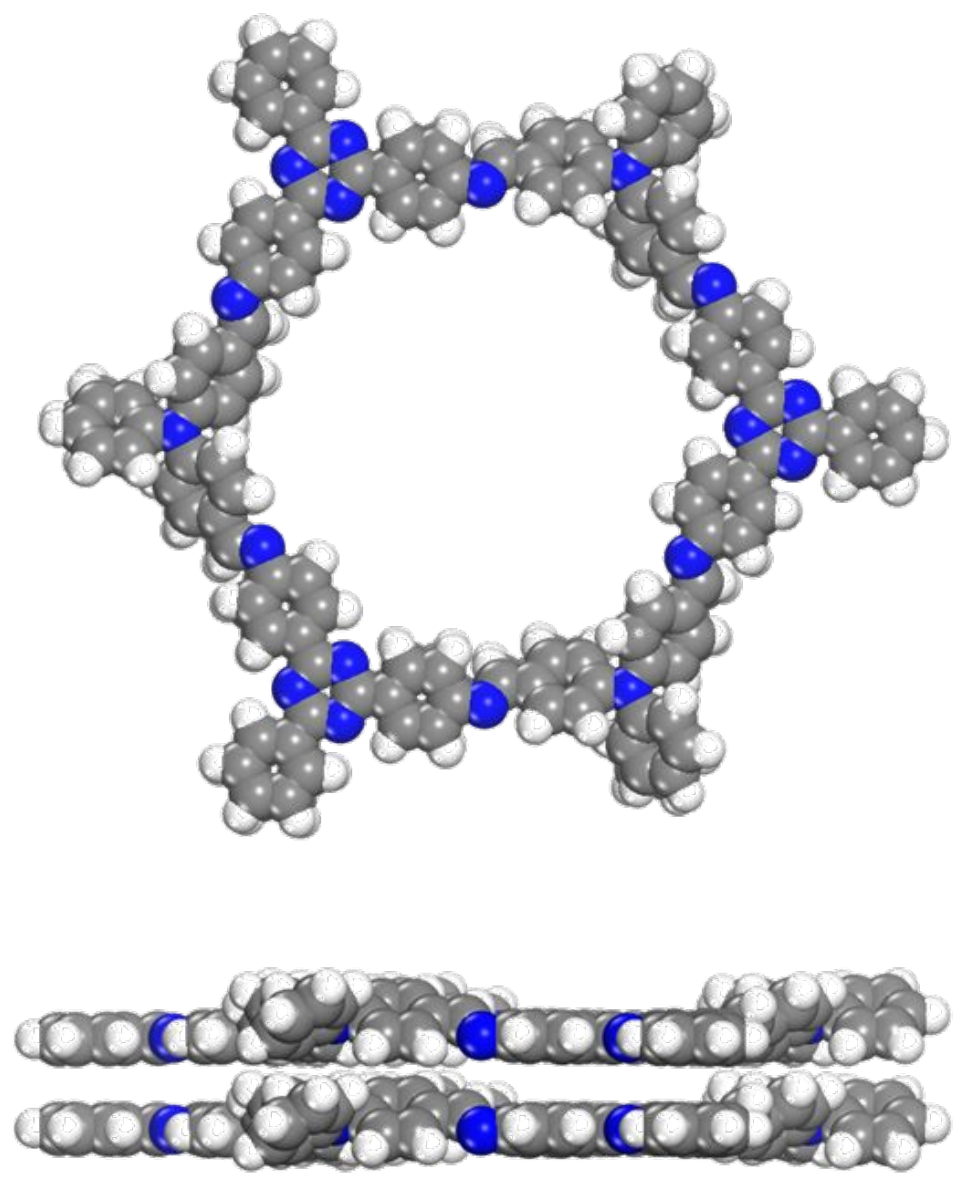

\section{Stacking energy per hexagon bilayer is $385 \mathrm{~kJ} / \mathrm{mol}$}

Supplementary Figure 21. Energy minimized hexagonally stacked bilayer (top and side views) of TAPT-TFPA COF based on DFT (Density Functional Theory). 

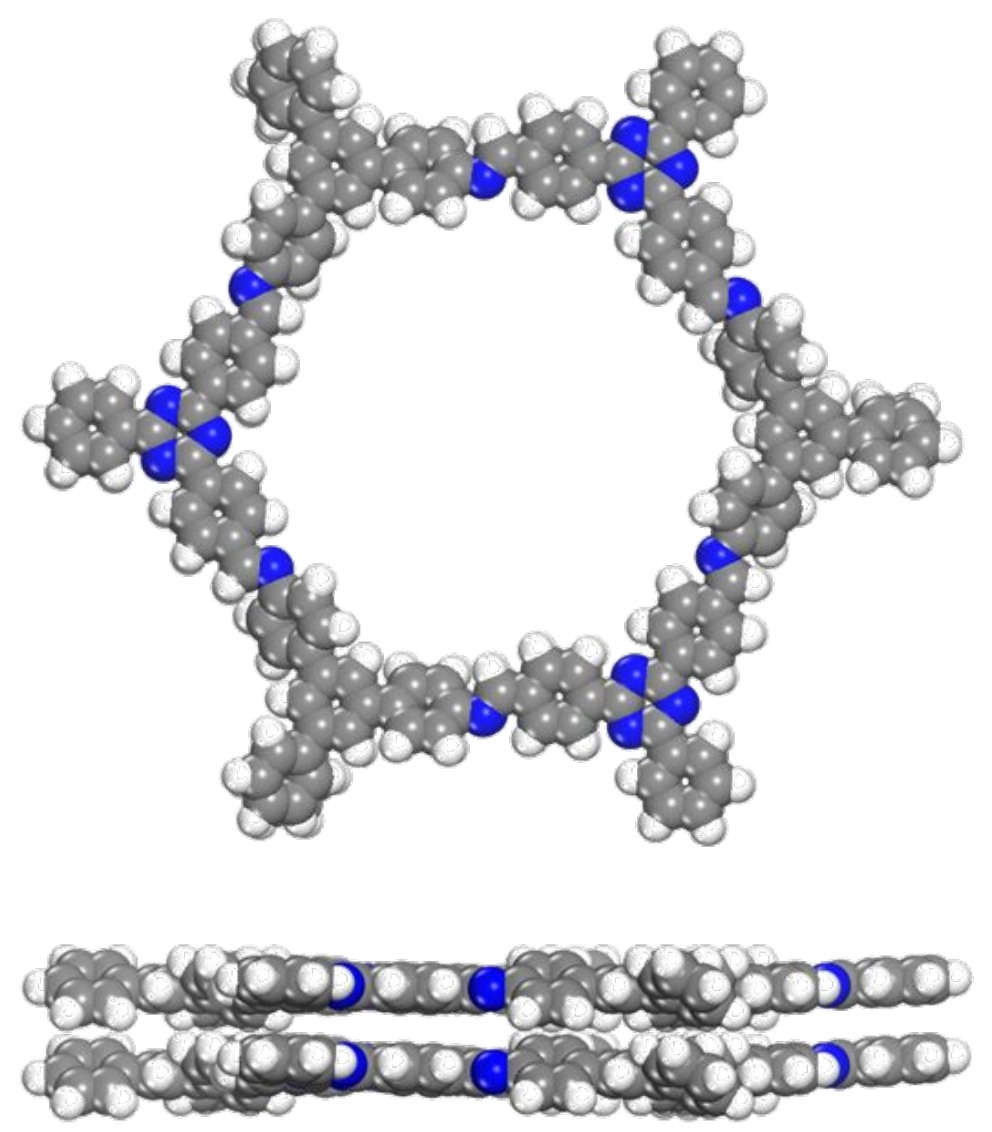

\section{Stacking energy per hexagon bilayer is $515 \mathrm{~kJ} / \mathrm{mol}$}

Supplementary Figure 22. Energy minimized hexagonally stacked bilayer (top and side views) of TAPB-TFPT COF based on DFT (Density Functional Theory). 

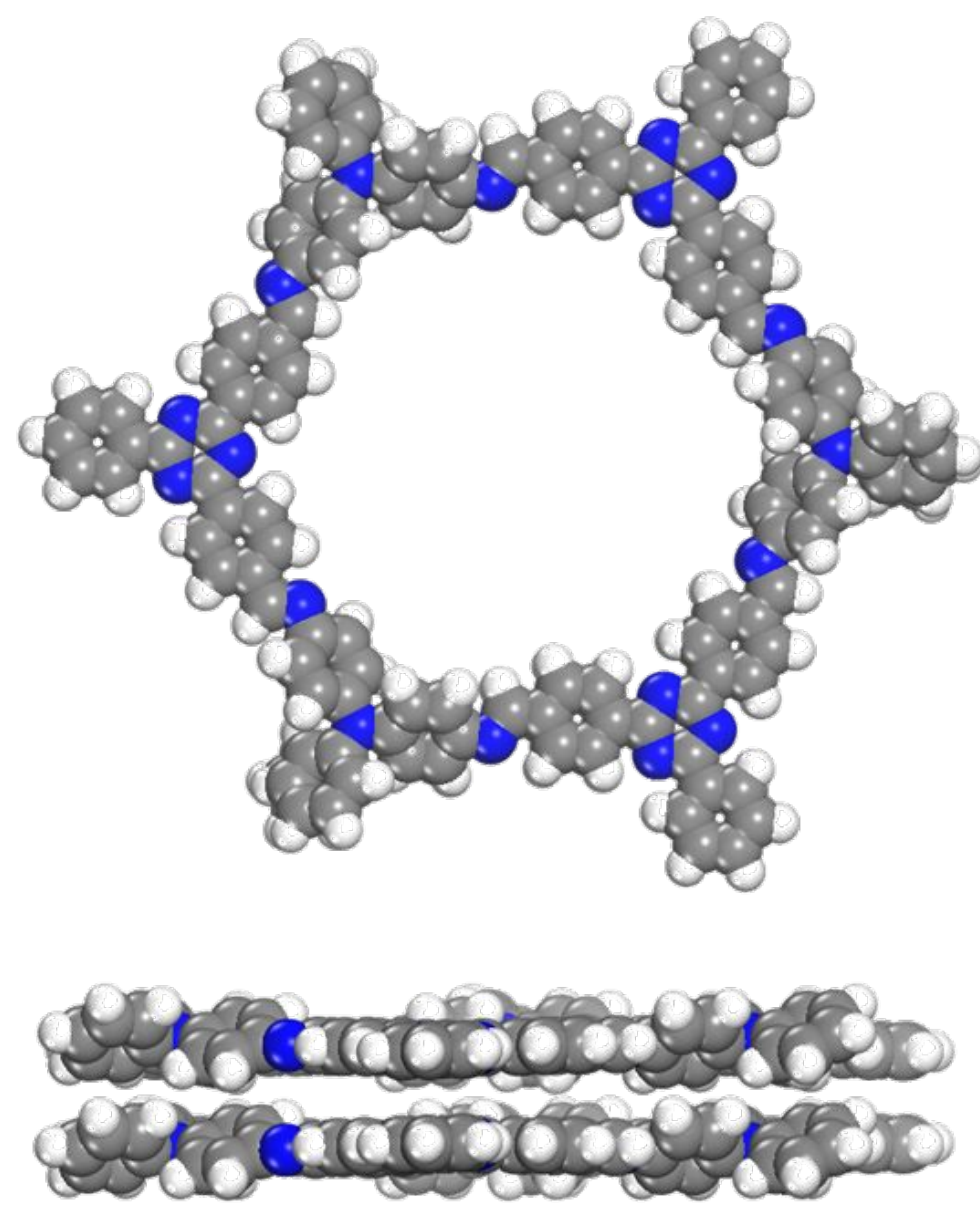

\section{Stacking energy per hexagon bilayer is $335 \mathrm{~kJ} / \mathrm{mol}$}

Supplementary Figure 23. Energy minimized hexagonally stacked bilayer (top and side views) of TAPA-TFPT COF based on DFT (Density Functional Theory). 

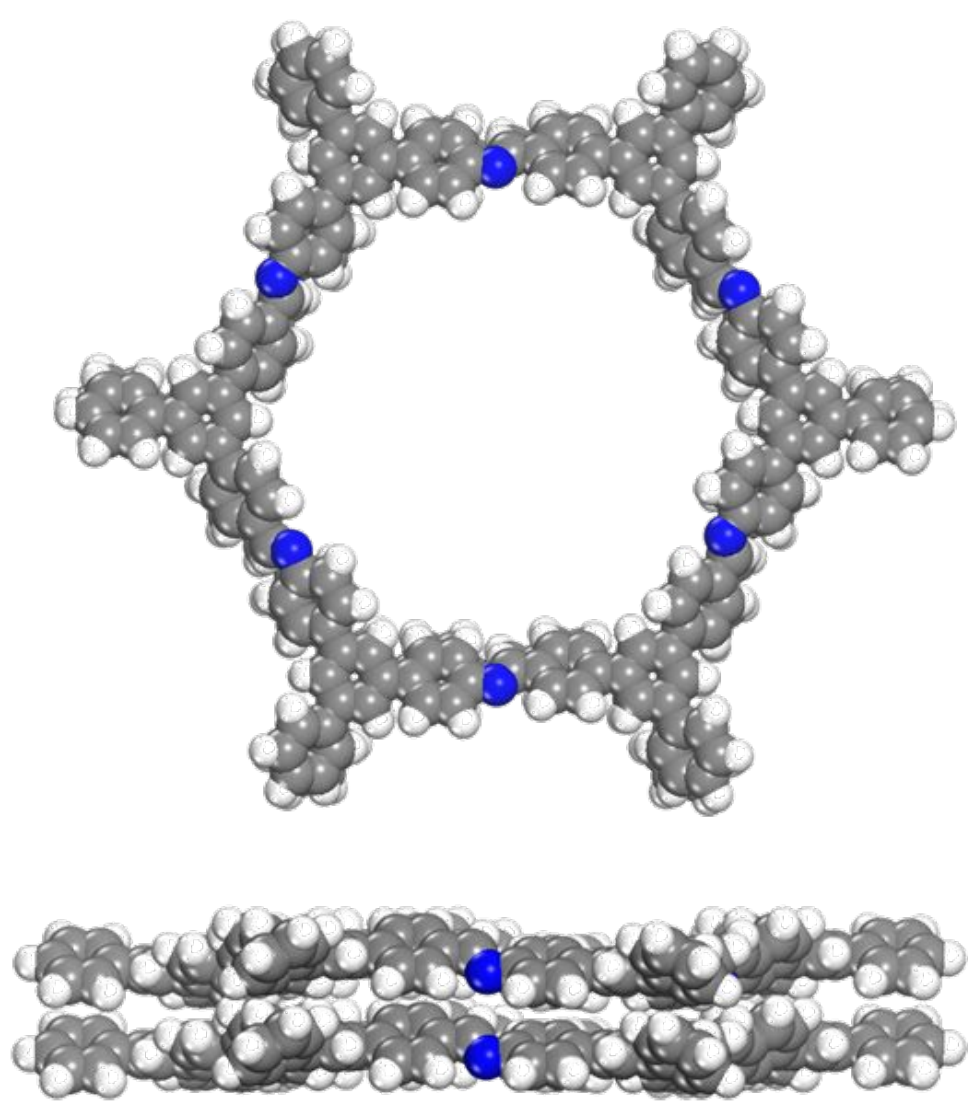

\section{Stacking energy per hexagon bilayer is $486 \mathrm{~kJ} / \mathrm{mol}$}

Supplementary Figure 24. Energy minimized hexagonally stacked bilayer (top and side views) of TAPB-TFPB COF based on DFT (Density Functional Theory). 

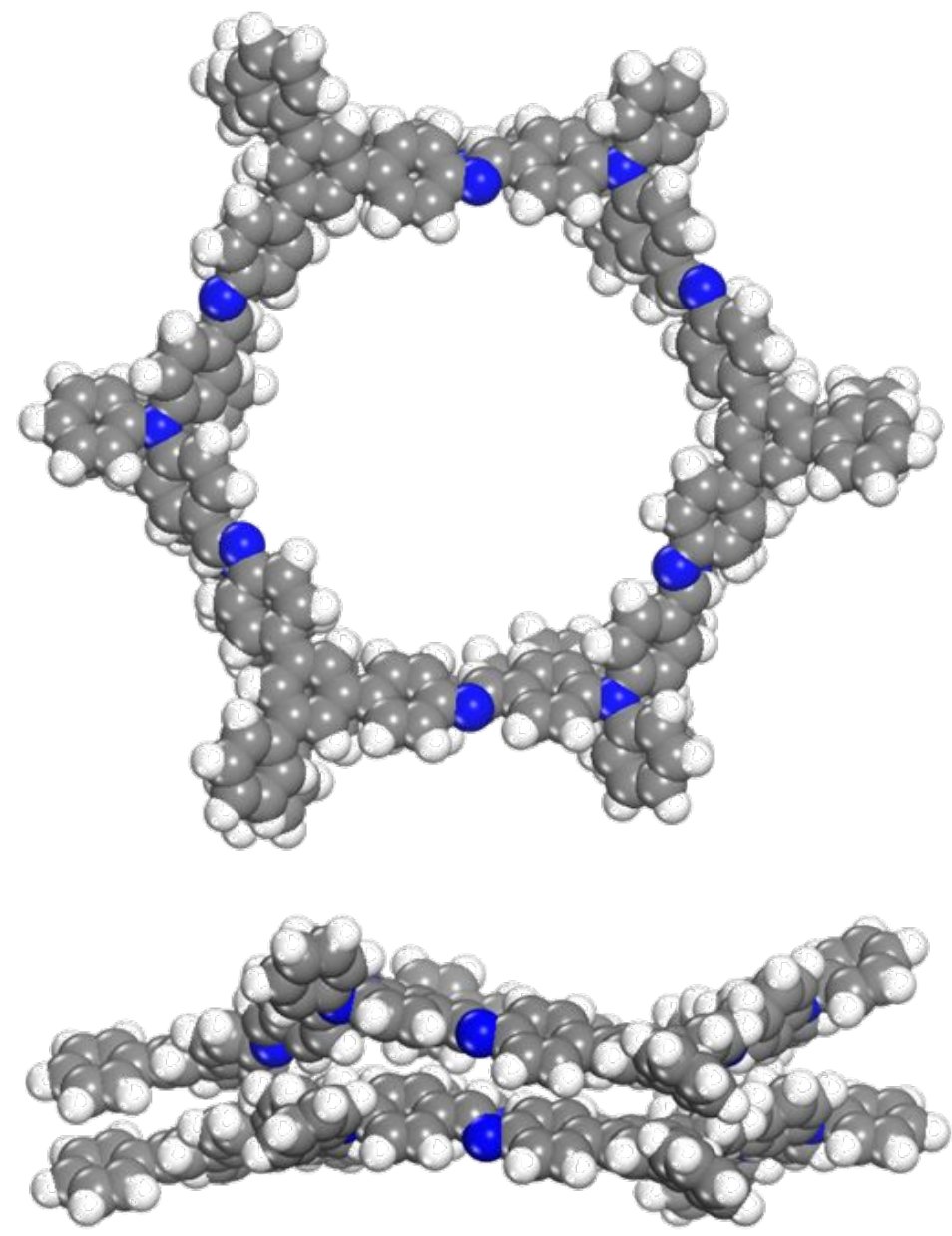

\section{Stacking energy per hexagon bilayer is $352 \mathrm{~kJ} / \mathrm{mol}$}

Supplementary Figure 25. Energy minimized hexagonally stacked bilayer (top and side views) of TAPB-TFPA COF based on DFT (Density Functional Theory). 

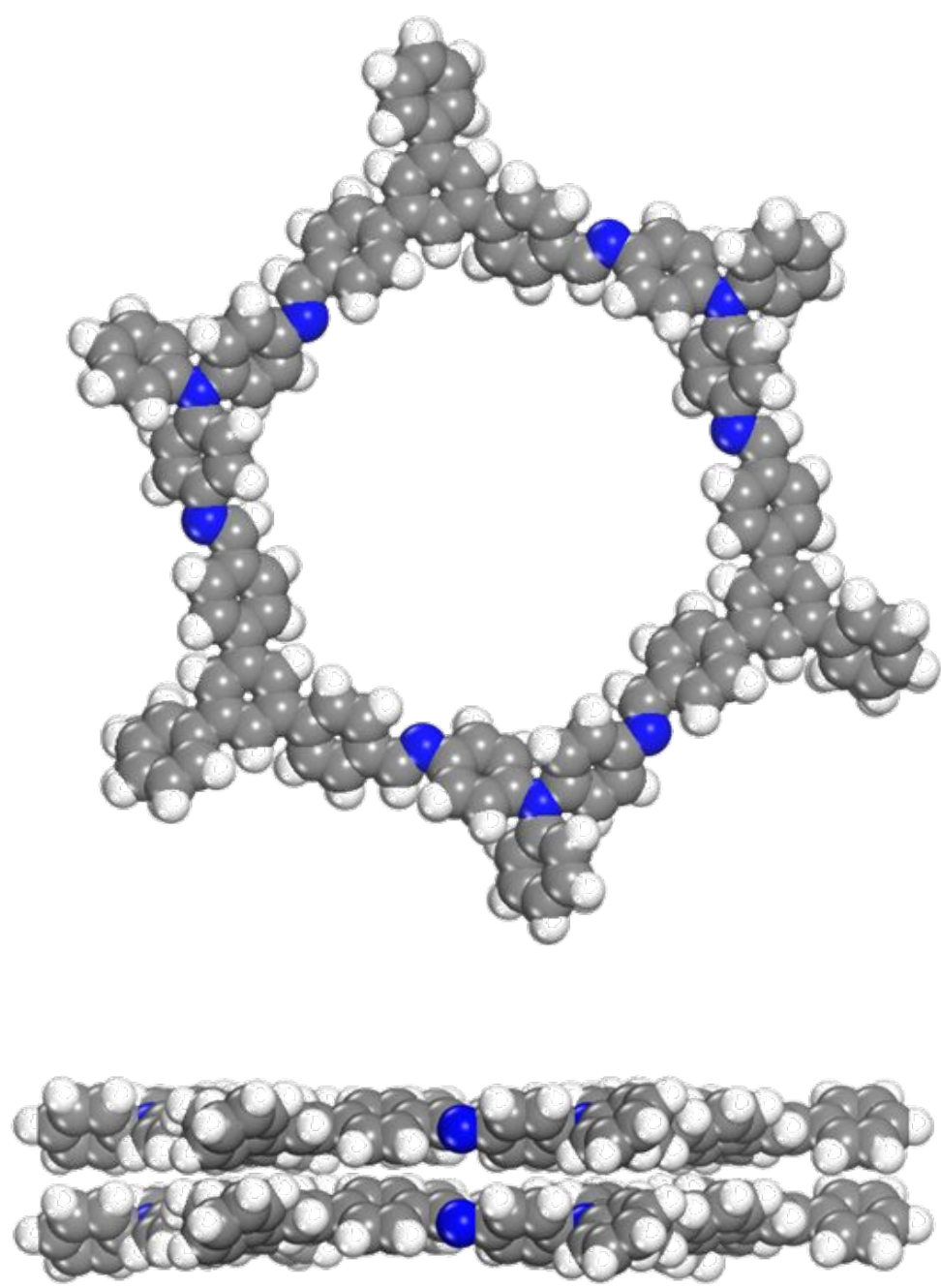

\section{Stacking energy per hexagon bilayer is $368 \mathrm{~kJ} / \mathrm{mol}$}

Supplementary Figure 26. Energy minimized hexagonally stacked bilayer (top and side views) of TAPA-TFPB COF based on DFT (Density Functional Theory). 

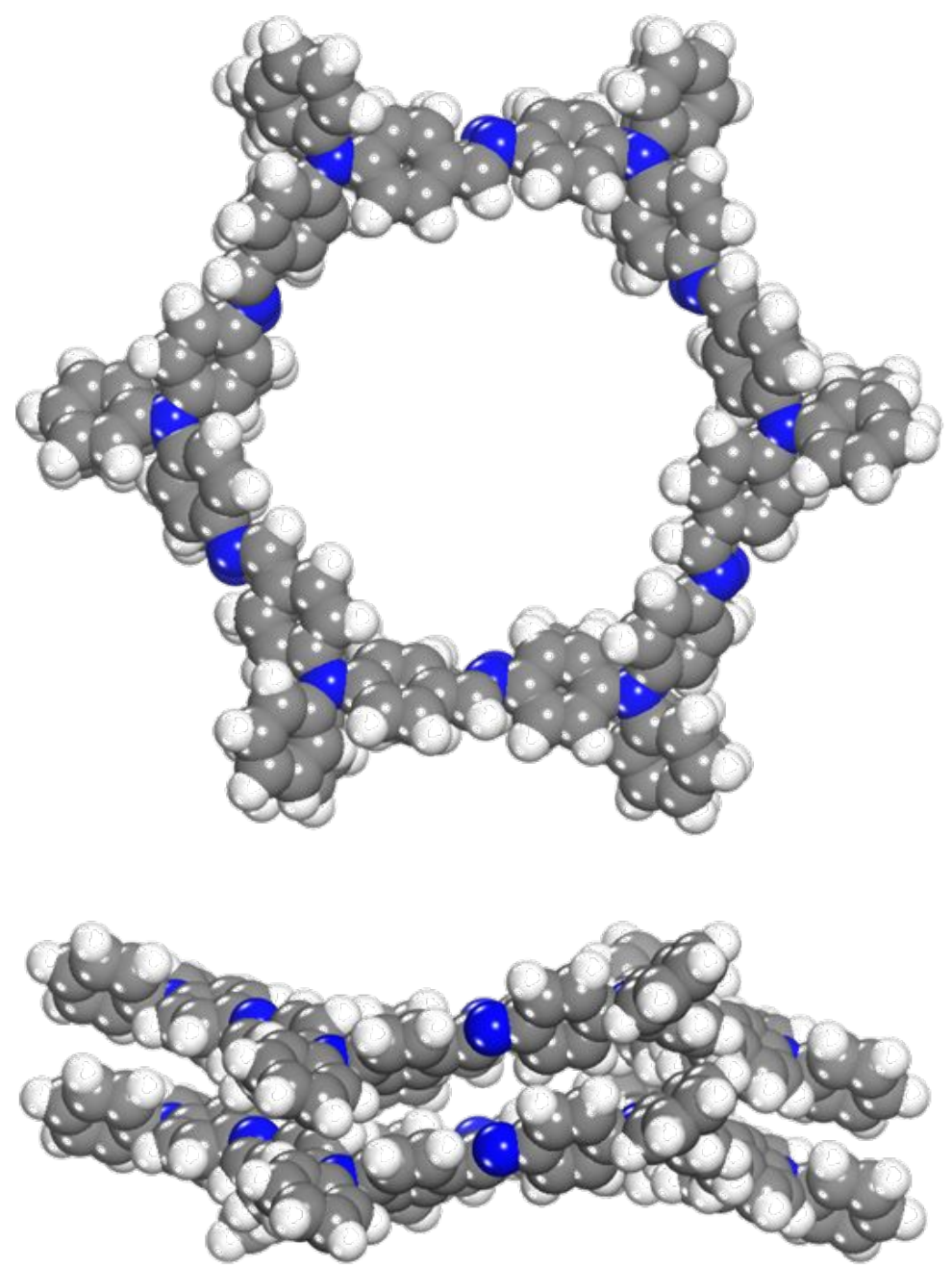

\section{Stacking energy per hexagon bilayer is $285 \mathrm{~kJ} / \mathrm{mol}$}

Supplementary Figure 27. Energy minimized hexagonally stacked bilayer (top and side views) of TAPA-TFPA COF based on DFT (Density Functional Theory). 


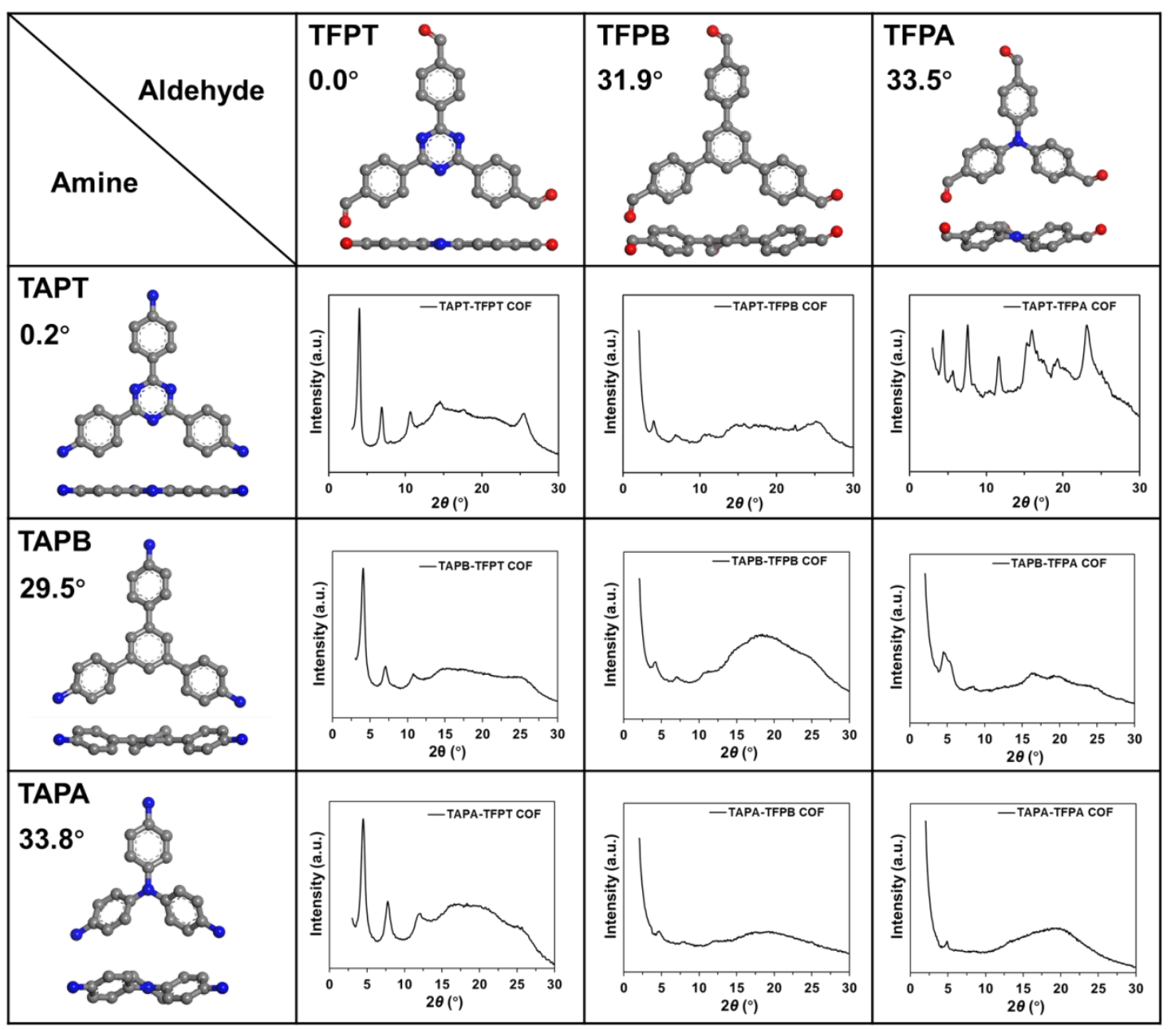

Supplementary Figure 28. PXRD spectra of different COFs washed by dioxane/mesitylene $(2 / 3, \mathrm{v} / \mathrm{v})$ and dry under vacuum at $50{ }^{\circ} \mathrm{C}$. 


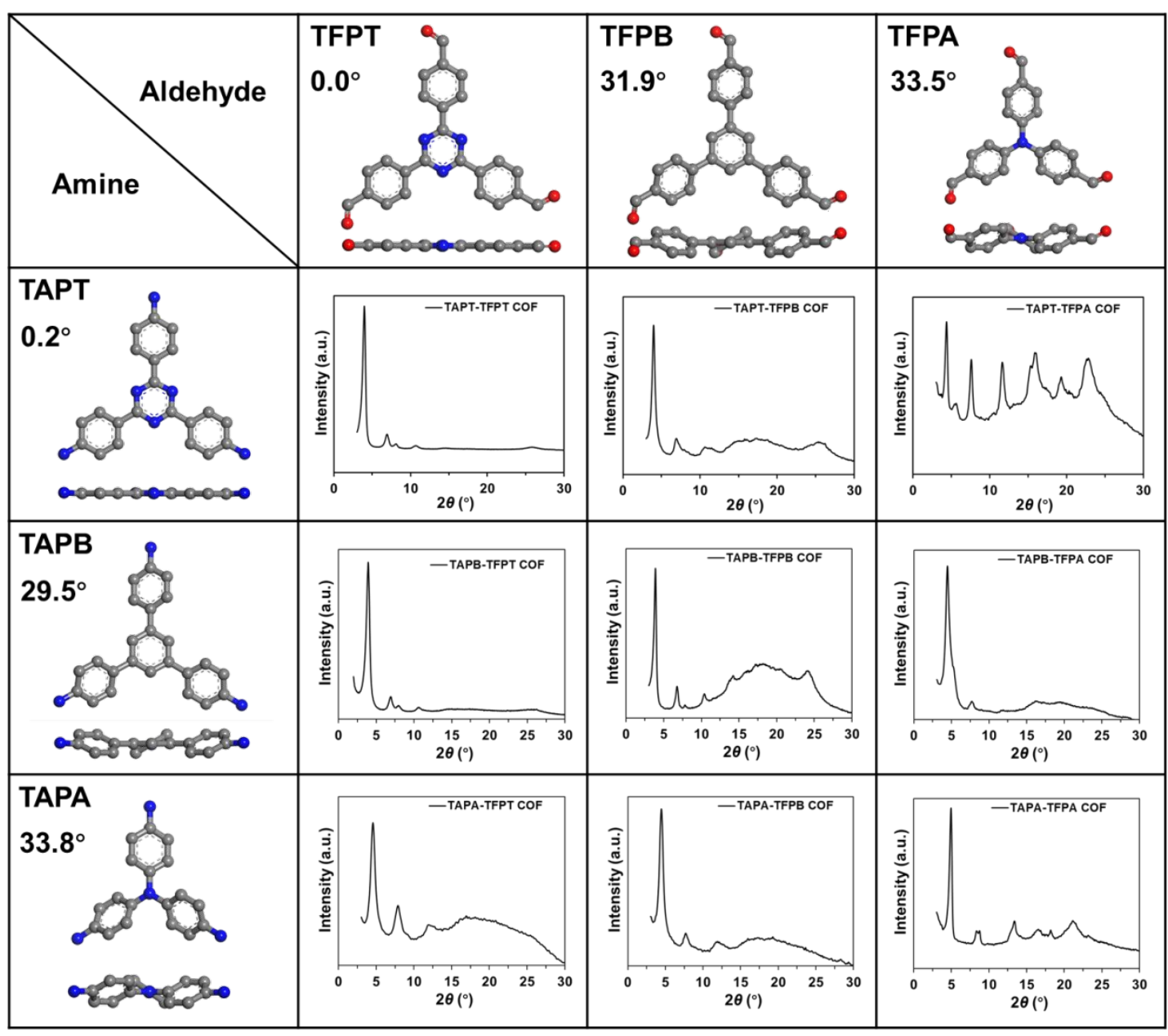

Supplementary Figure 29. PXRD spectra of different COFs washed by hexane and dry in the ambient. Besides morphologies, tolerance for pore collapse under the capillary force during solvent evaporation ${ }^{3-5}$ is also different for these COFs. We first use dioxane/mesitylene $(2 / 3, v / v)$, the synthetic solvent to wash the products and dry directly under vacuum at $50^{\circ} \mathrm{C}$. High planar COFs, TAPT-TFPT, TAPB-TFPT, TAPATFPT and TAPT-TFPA COFs maintain good crystallinity, evidenced by sharp diffraction peaks of PXRD spectra (Supplementary Fig. 28). The pore structures of the low planar COFs collapse with poor diffraction peaks (Supplementary Fig. 28) after the drying process. This can be explained that high interlayer reaction will can overcome the capillary force and maintain the honeycomb crystalline structures. Then, we use low surface tension solvent, i.e. hexane to wash the produces and dry slowly in the ambient. Such drying process dramatically avoids pore collapse and show intrinsic crystallinity of COFs by minimizing the capillary force. As a result, all the COFs show sharp diffraction peaks of PXRD spectra (Supplementary Fig. 29). 

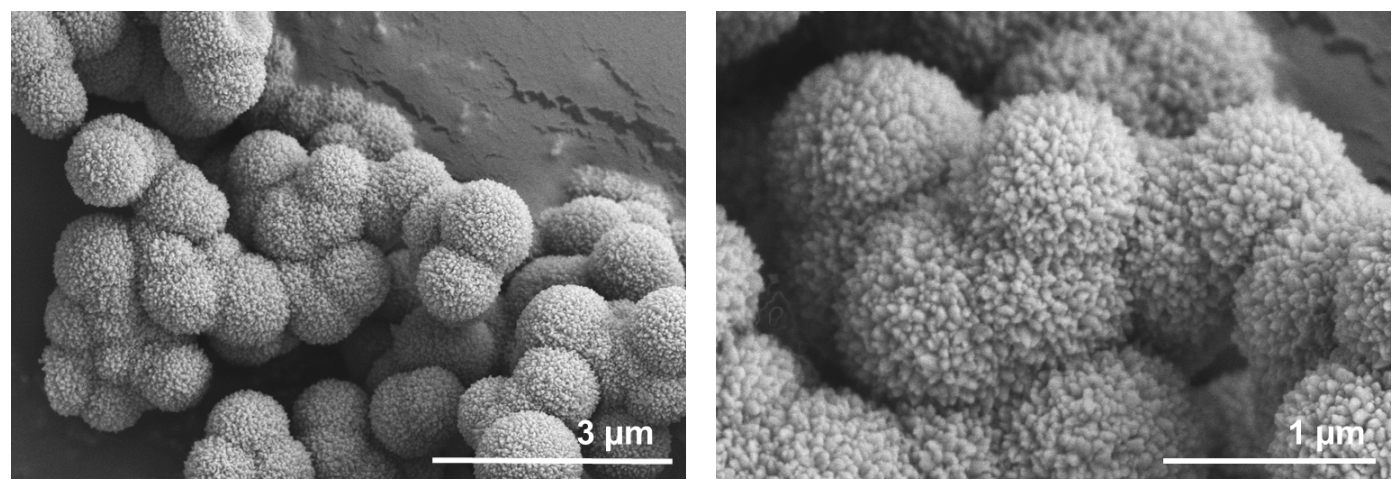

Supplementary Figure 30. FESEM images of TAPT-TFPT COF.
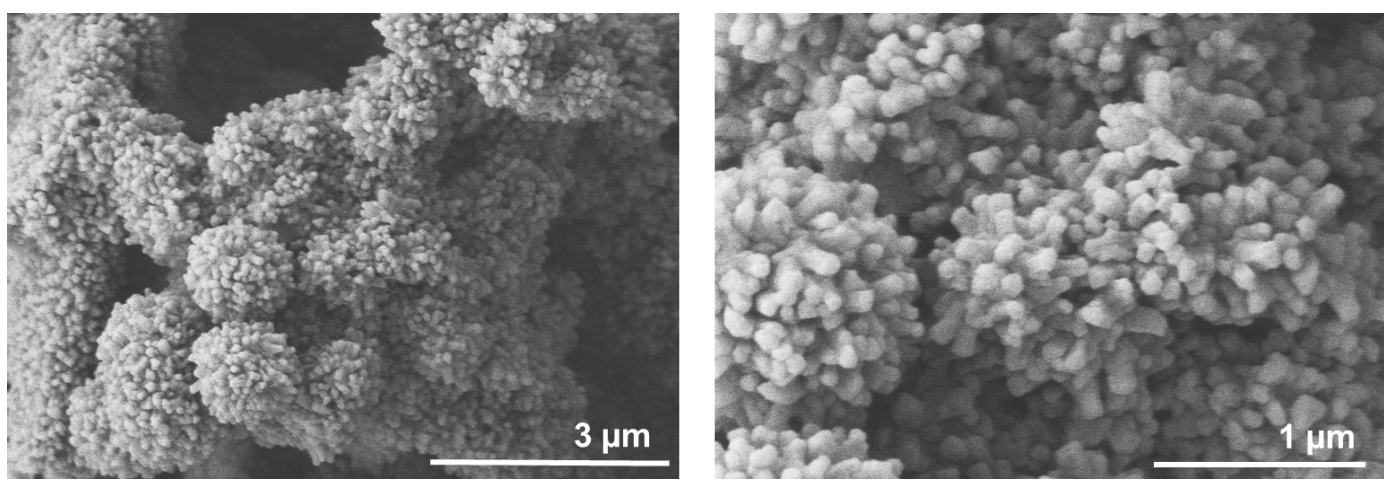

Supplementary Figure 31. FESEM images of TAPT-TFPB COF.
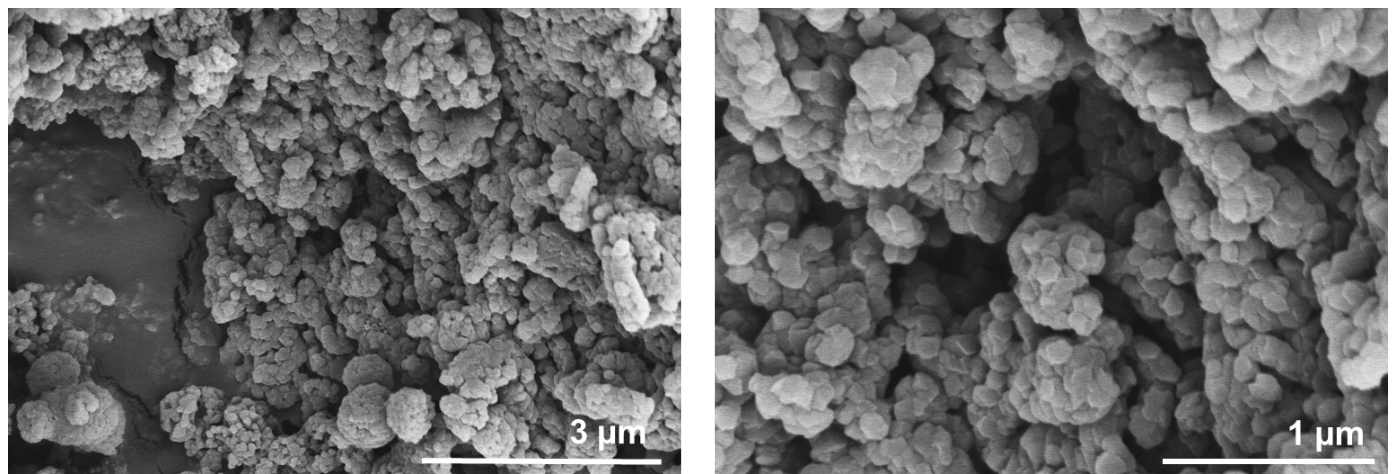

Supplementary Figure 32. FESEM images of TAPT-TFPA COF. 

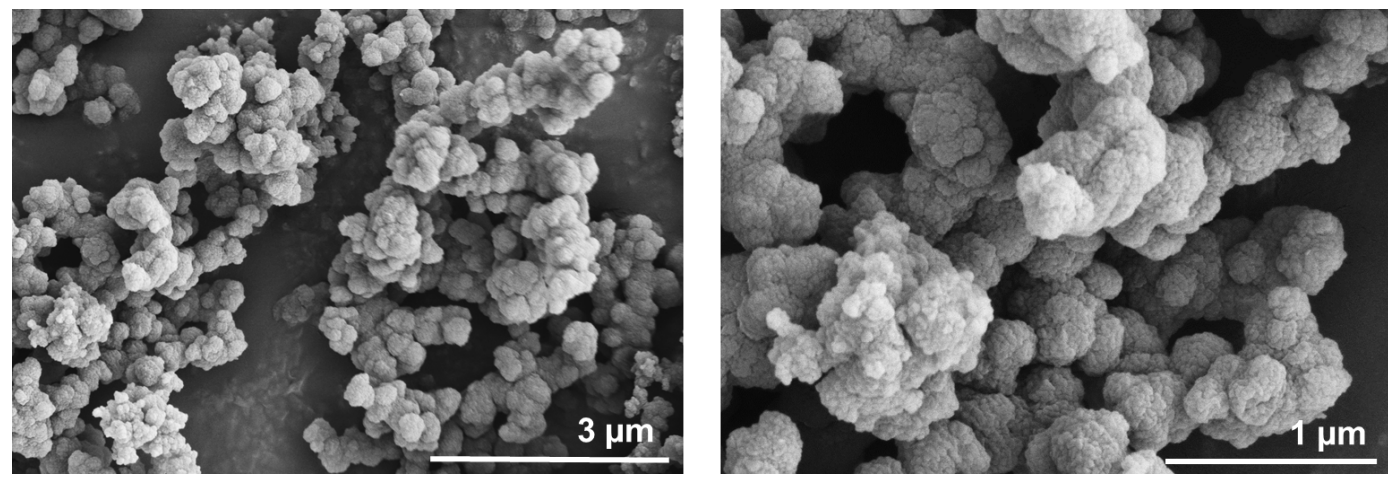

Supplementary Figure 33. FESEM images of TAPB-TFPT COF.
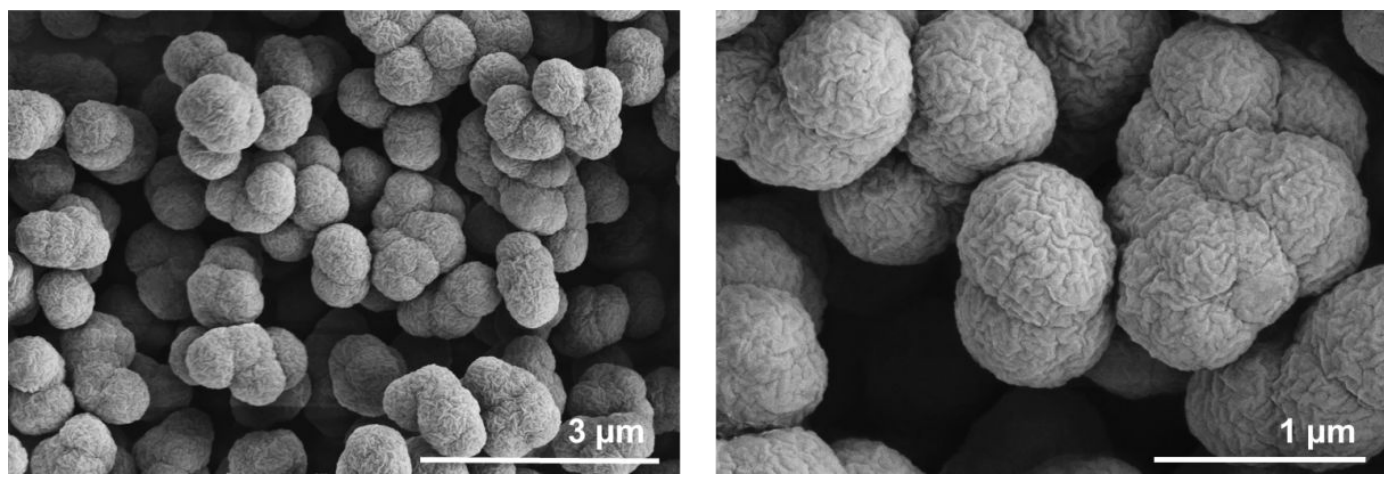

Supplementary Figure 34. FESEM images of TAPB-TFPB COF.
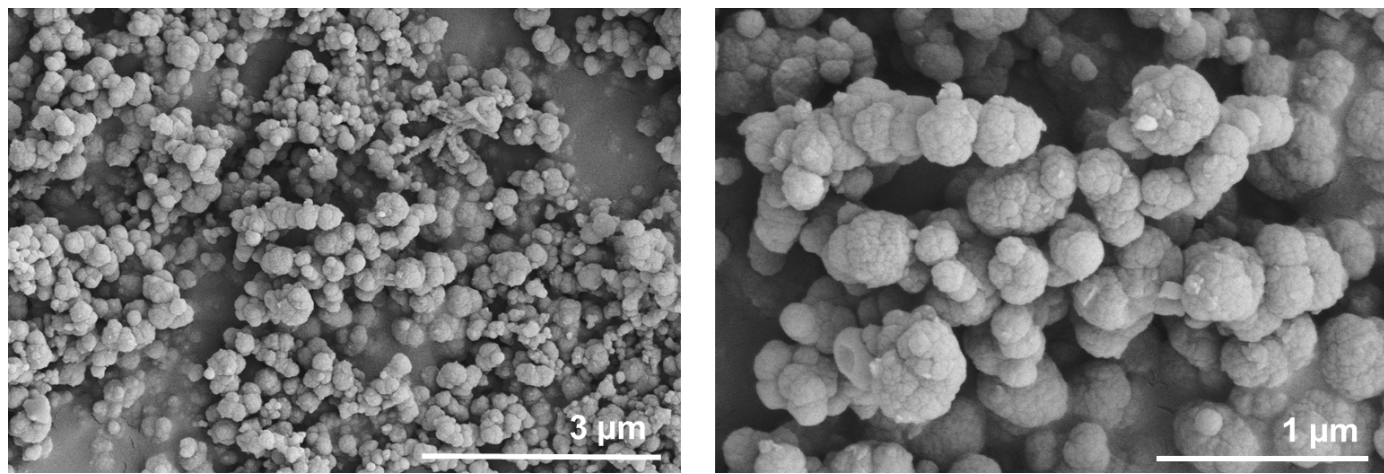

Supplementary Figure 35. FESEM images of TAPA-TFPT COF. 

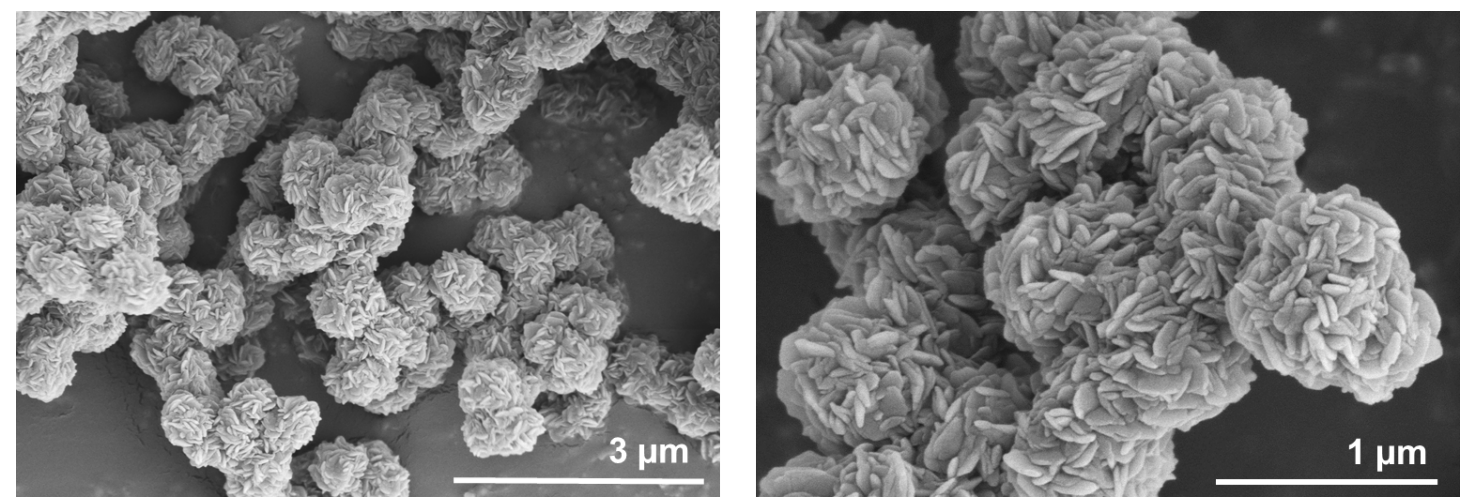

Supplementary Figure 36. FESEM images of TAPB-TFPA COF.
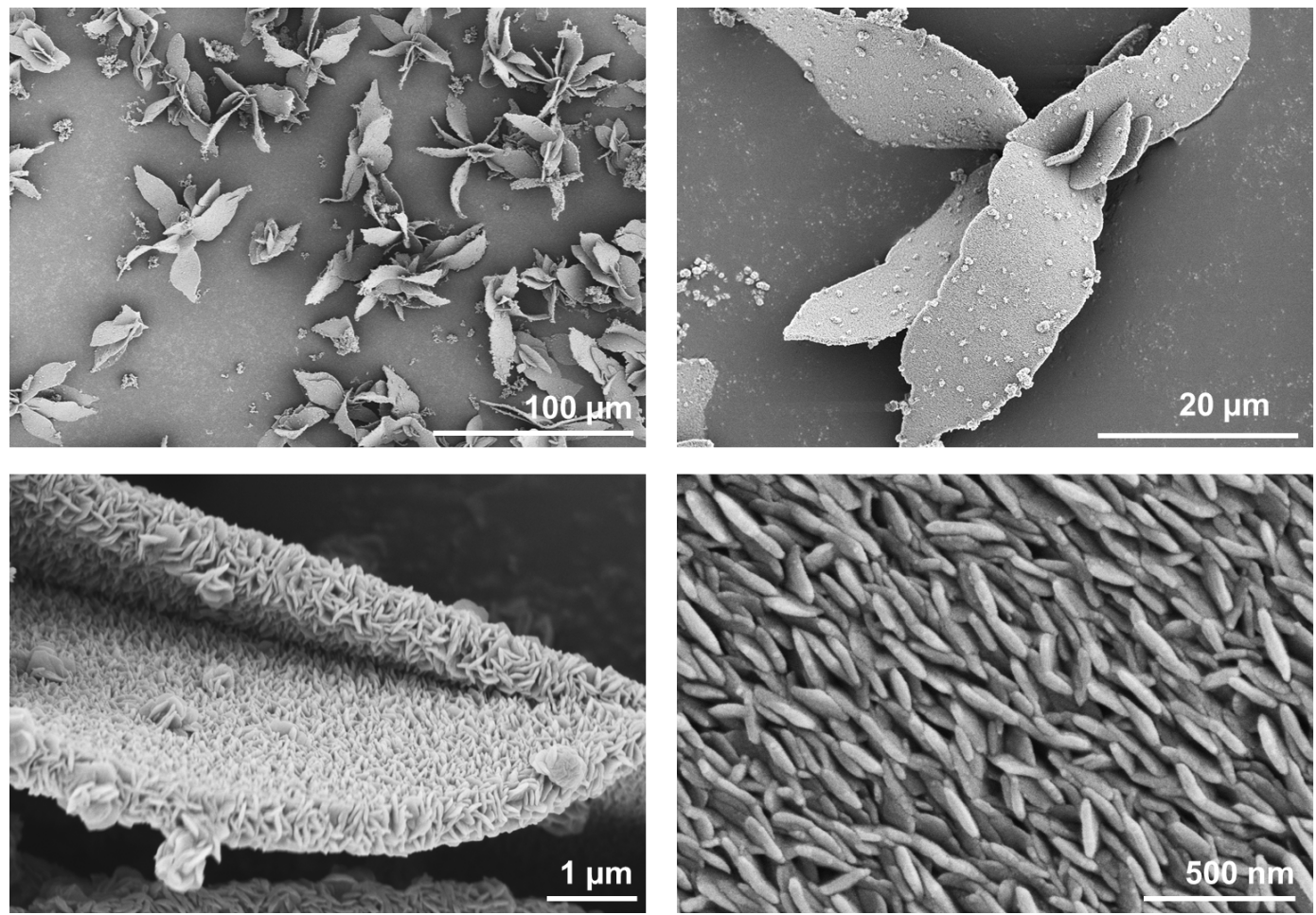

Supplementary Figure 37. FESEM images of TAPA-TFPB COF. 

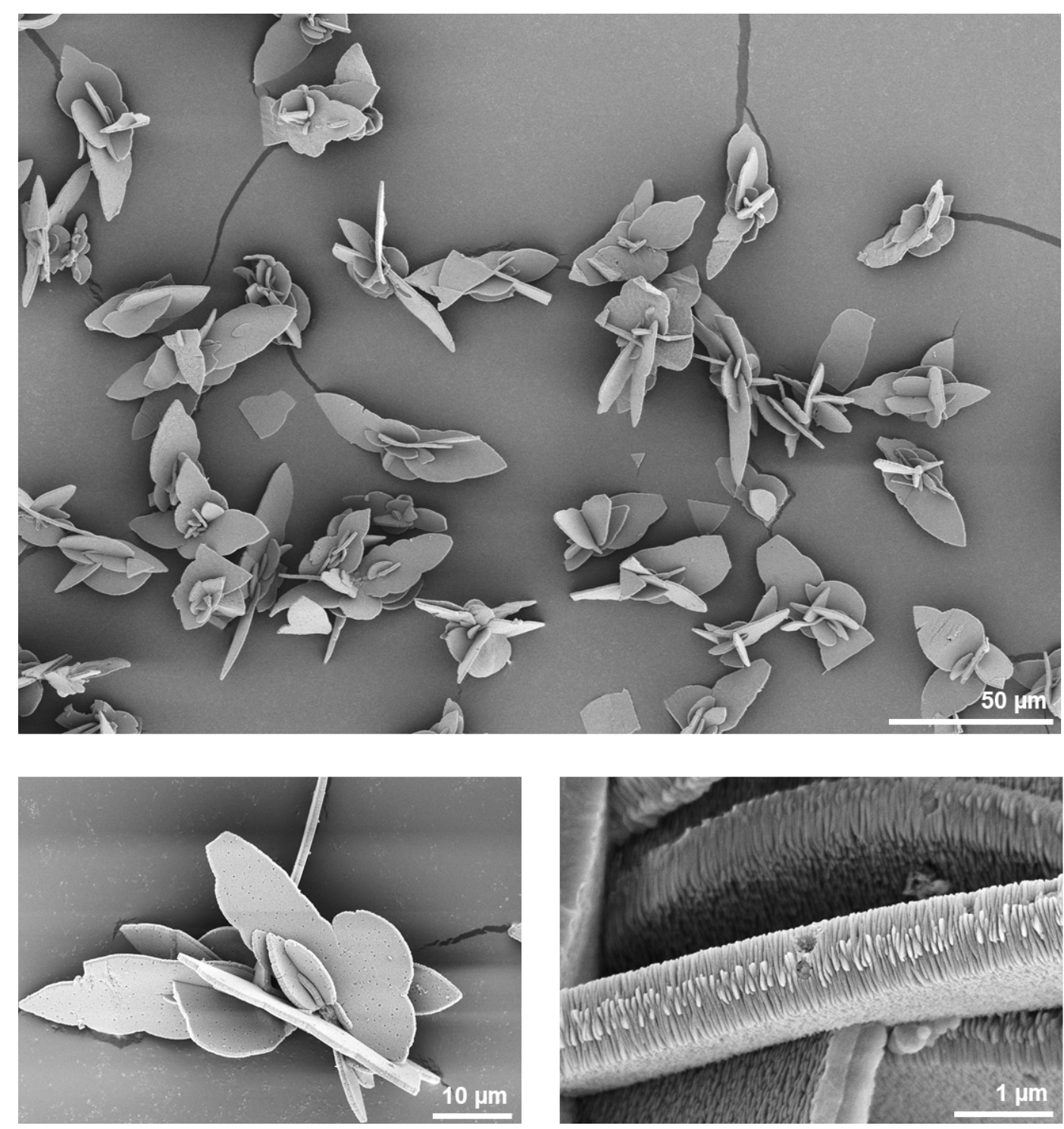

Supplementary Figure 38. FESEM images of the TAPA-TFPA COF flower synthesized at $60{ }^{\circ} \mathrm{C}$ in different scales. 

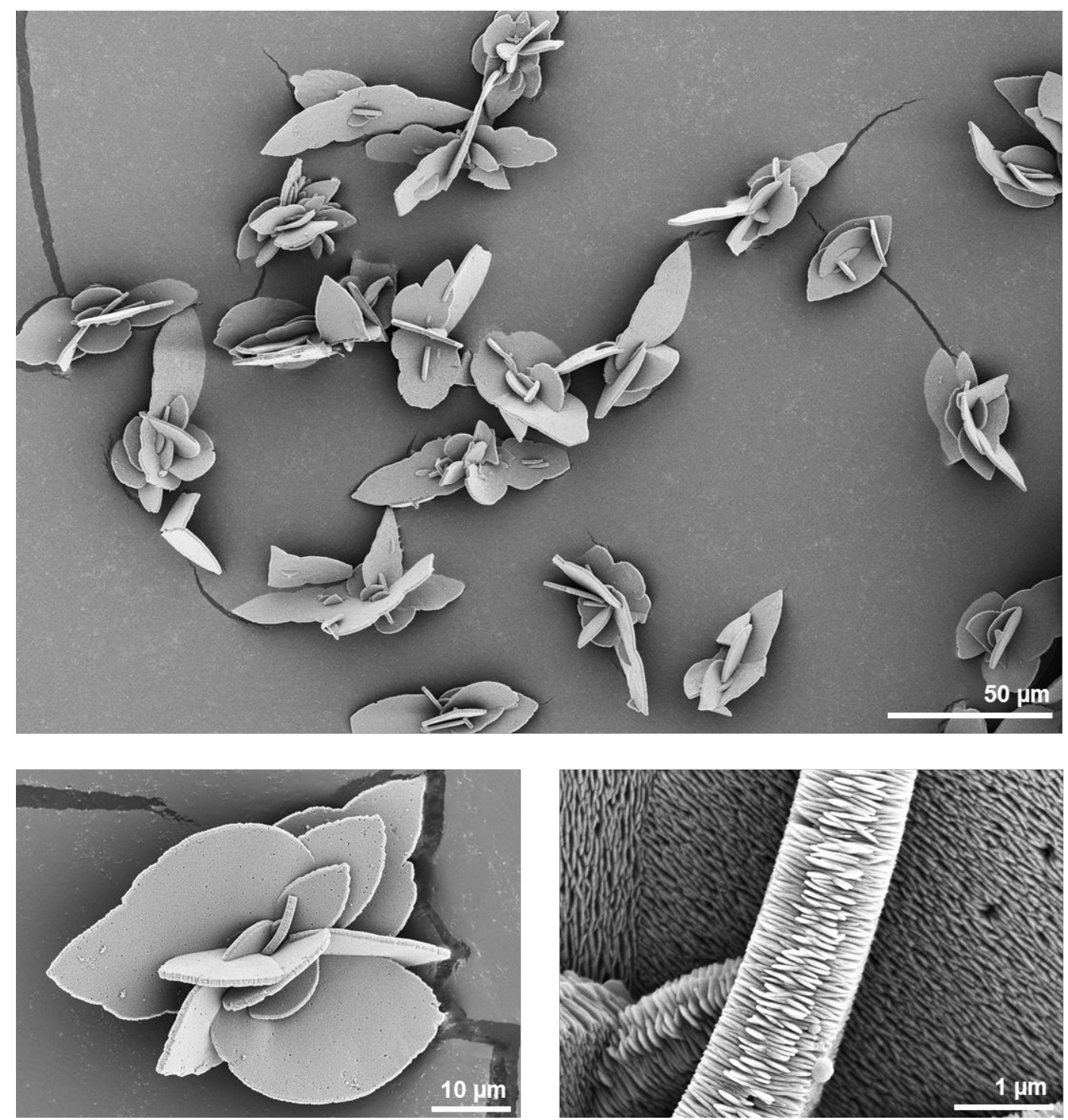

Supplementary Figure 39. FESEM images of the TAPA-TFPA COF flower synthesized at $80^{\circ} \mathrm{C}$ in different scales. 

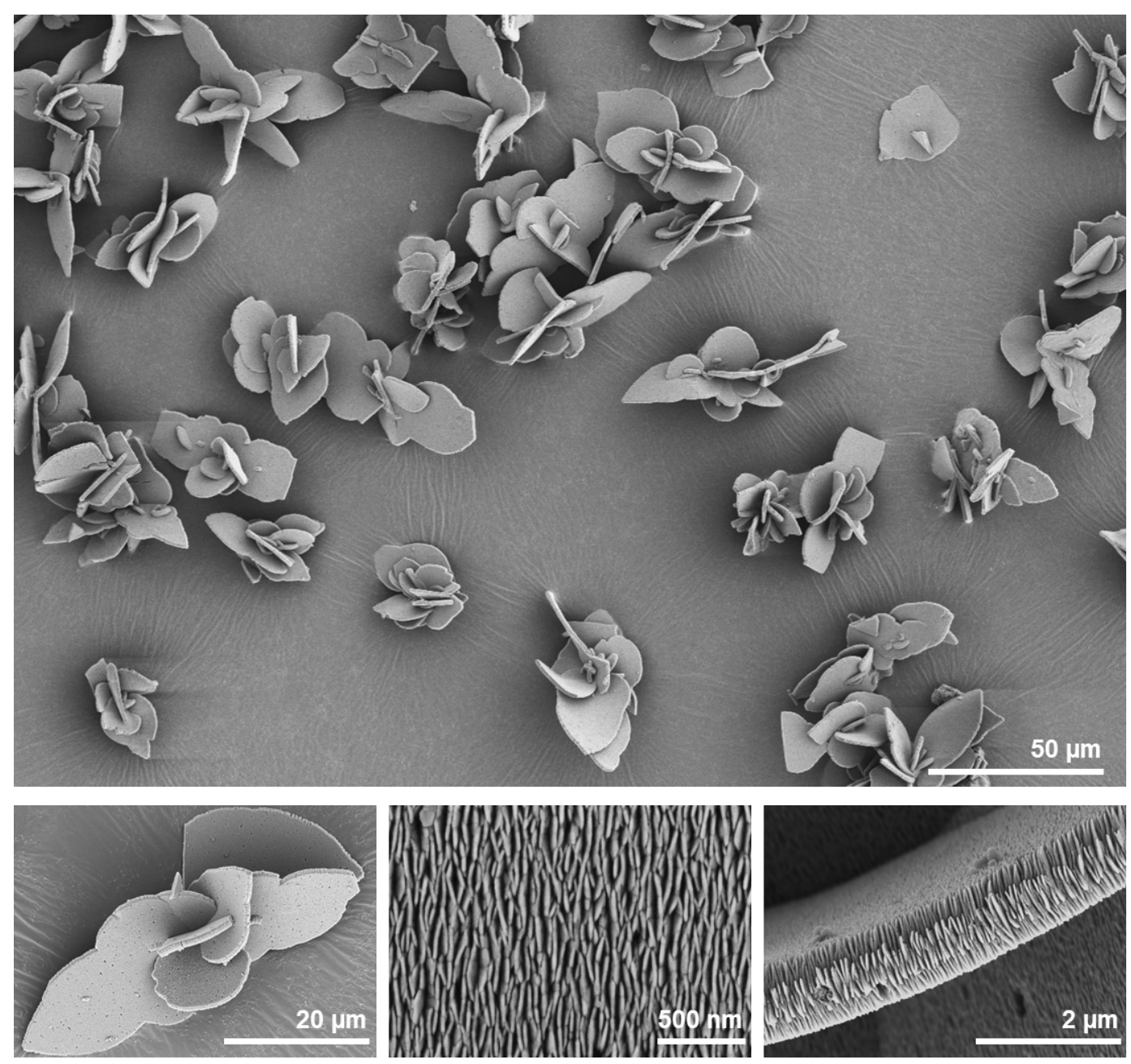

Supplementary Figure 40. FESEM images of the TAPA-TFPA COF flower synthesized at $100{ }^{\circ} \mathrm{C}$ in different scales. 


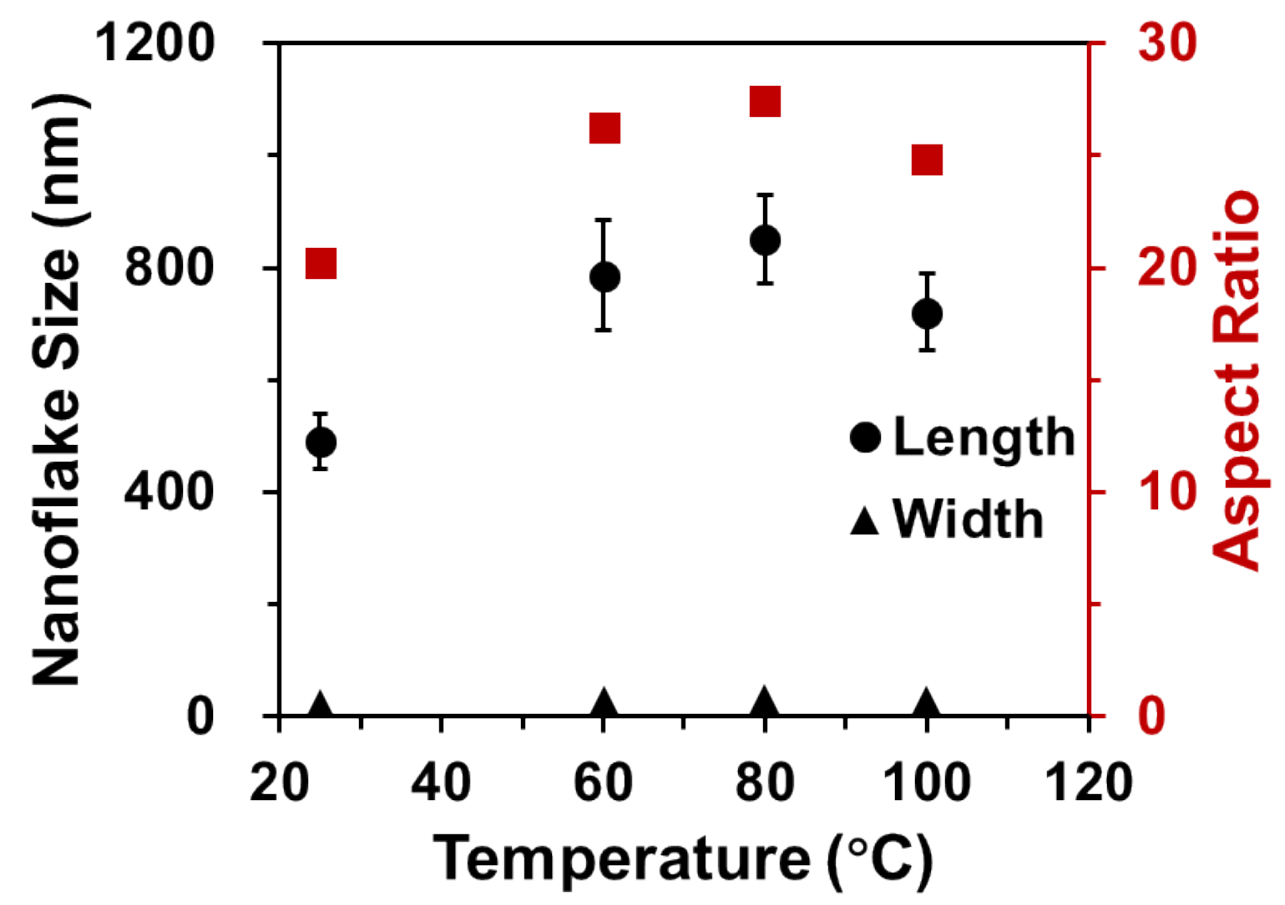

Supplementary Figure 41. Size and aspect ratio of nanoflakes on COF flowers synthesized at different temperatures.

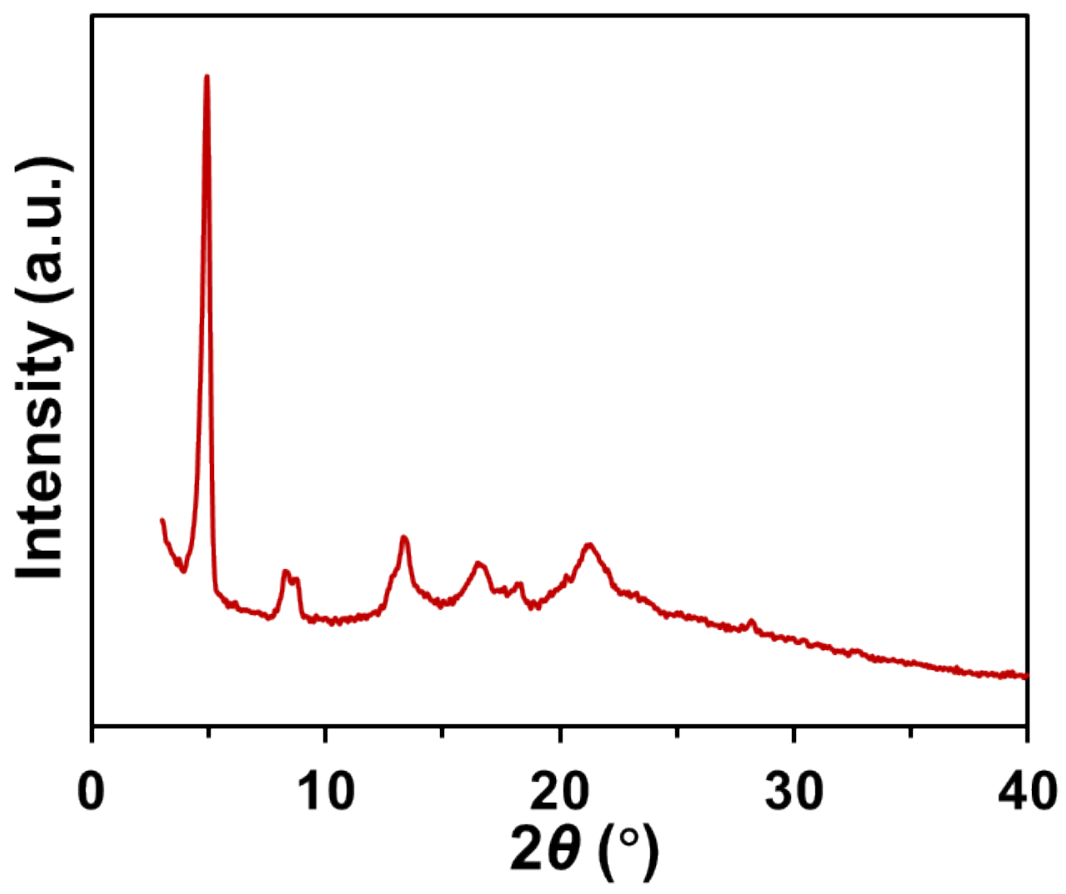

Supplementary Figure 42. PXRD diffraction pattern of TAPA-TFPA COF flower synthesized at $60{ }^{\circ} \mathrm{C}$. 


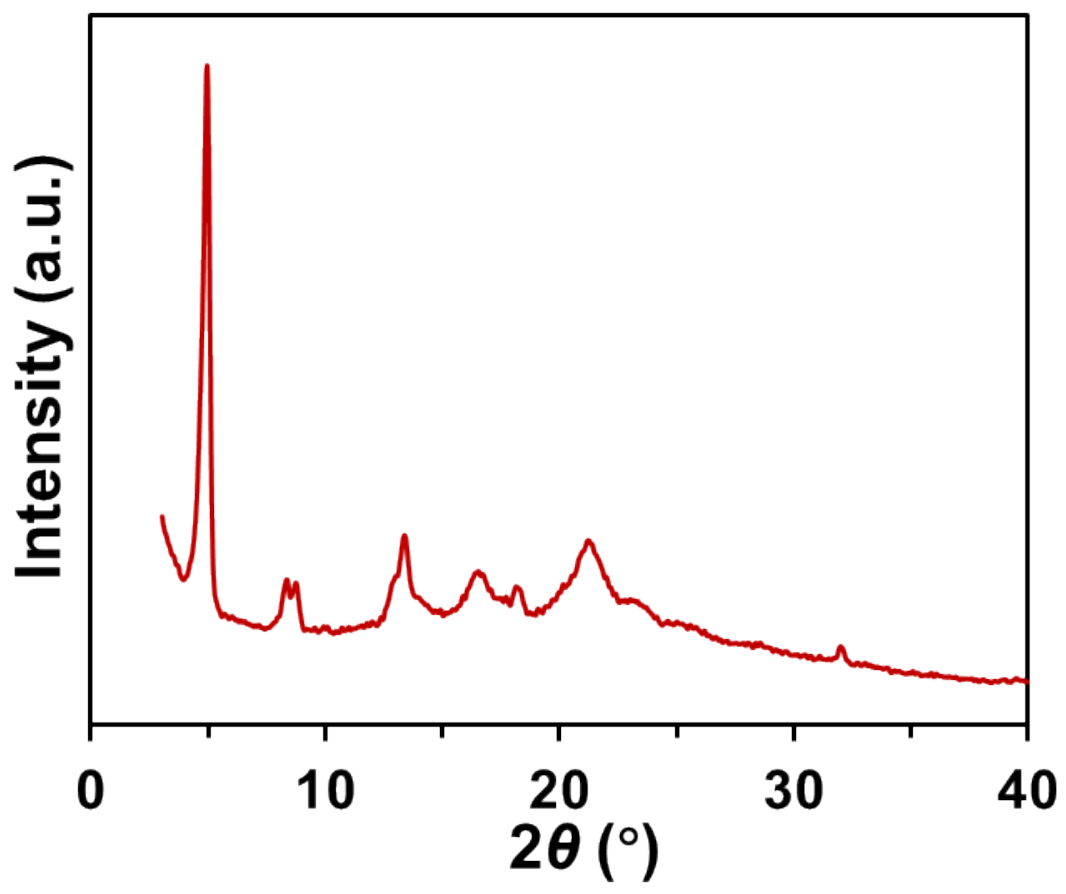

Supplementary Figure 43. PXRD diffraction pattern of TAPA-TFPA COF flower synthesized at $80^{\circ} \mathrm{C}$.

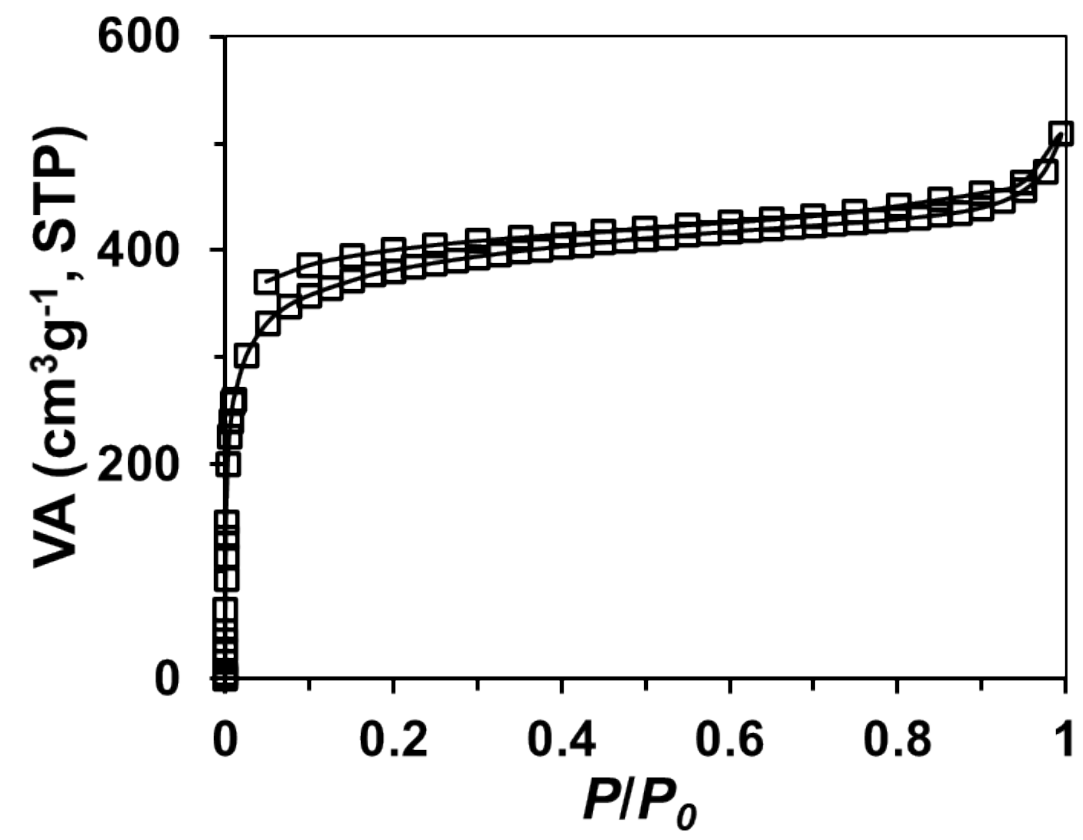

Supplementary Figure 44. Nitrogen sorption isotherm at $77 \mathrm{~K}$ for TAPA-TFPA COF flower synthesized at $60{ }^{\circ} \mathrm{C}$. 


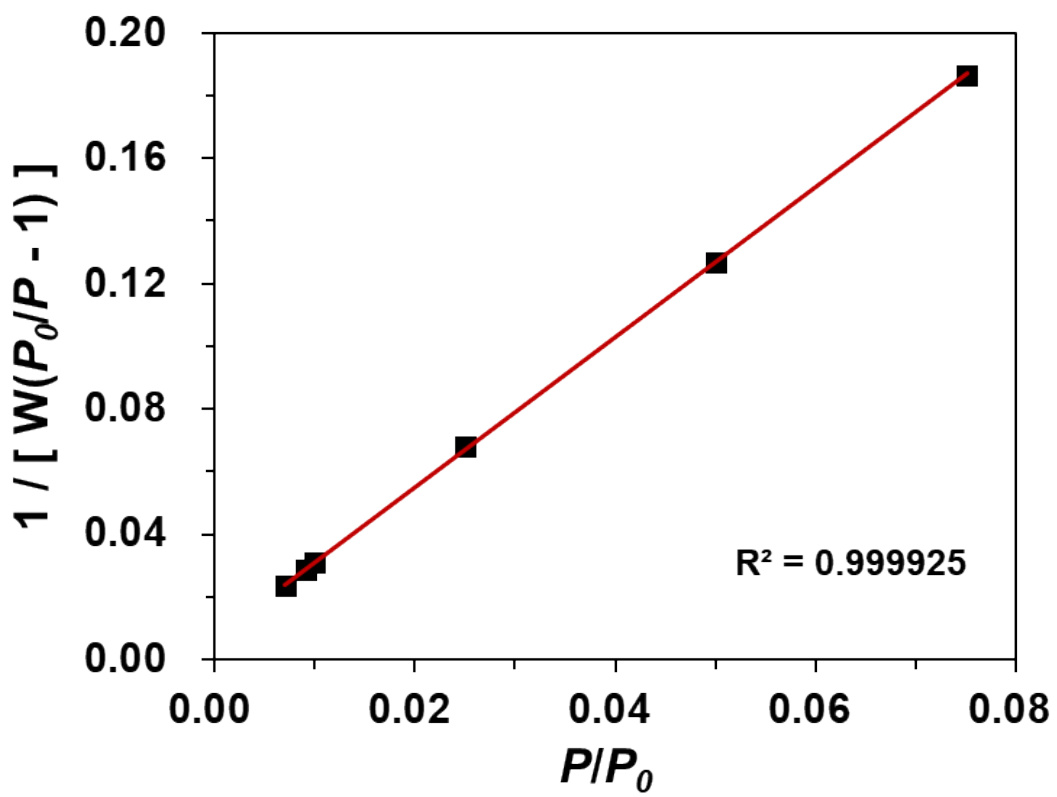

Supplementary Figure 45. BET plot for TAPA-TFPA COF flower synthesized at $60{ }^{\circ} \mathrm{C}$ calculated from nitrogen adsorption data.

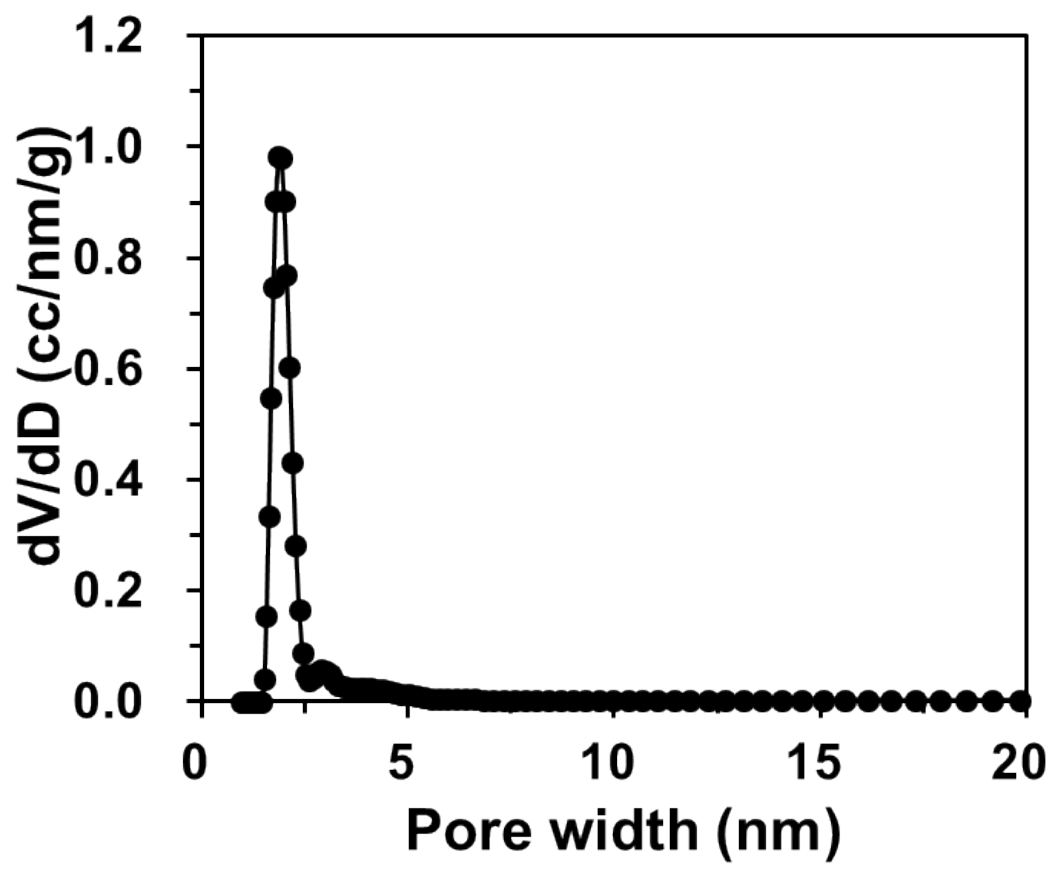

Supplementary Figure 46. Pore width distribution calculated by NLDFT model of TAPA-TFPA COF flower synthesized at $60^{\circ} \mathrm{C}$. 


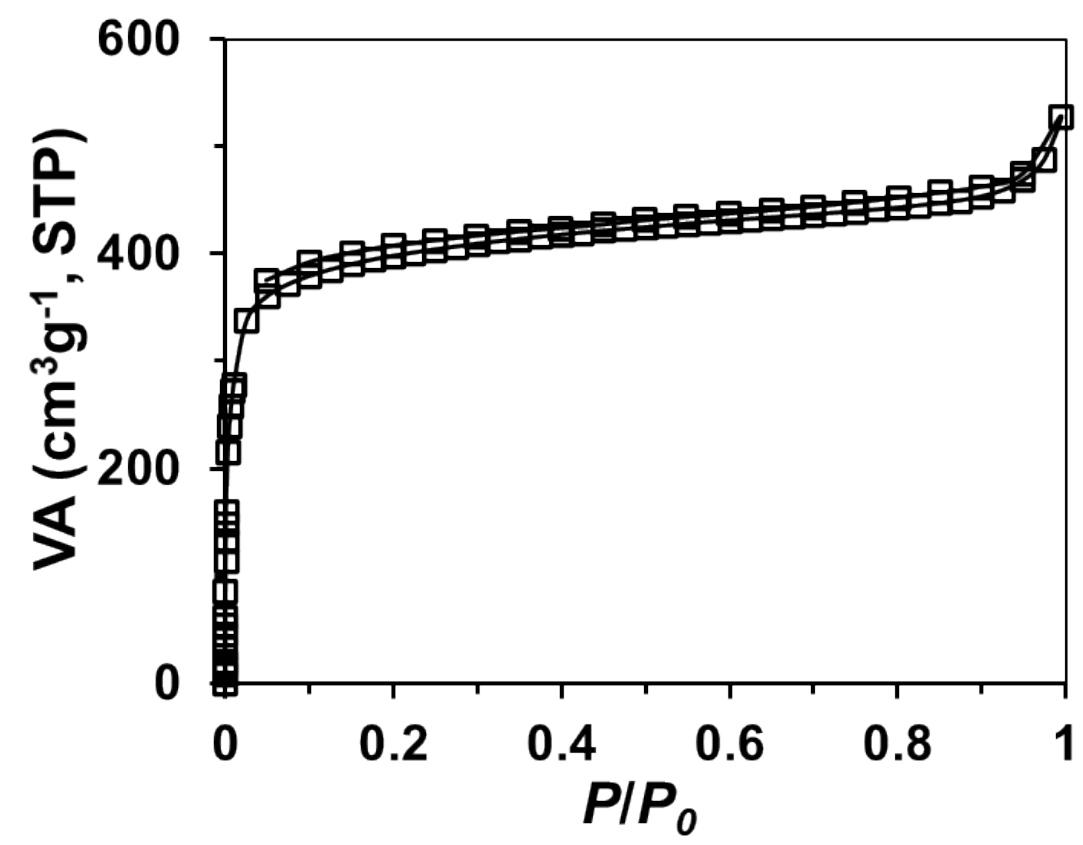

Supplementary Figure 47. Nitrogen sorption isotherm at $77 \mathrm{~K}$ for TAPA-TFPA COF flower synthesized at $80{ }^{\circ} \mathrm{C}$.

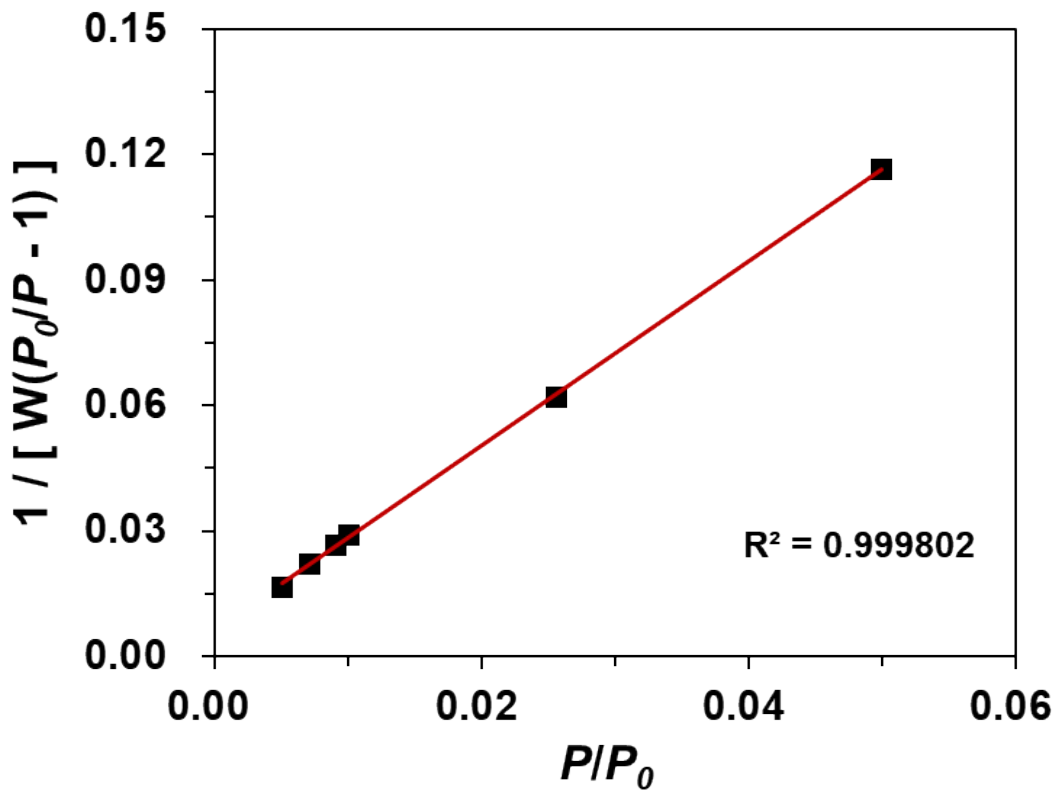

Supplementary Figure 48. BET plot for TAPA-TFPA COF flower synthesized at $80{ }^{\circ} \mathrm{C}$ calculated from nitrogen adsorption data. 


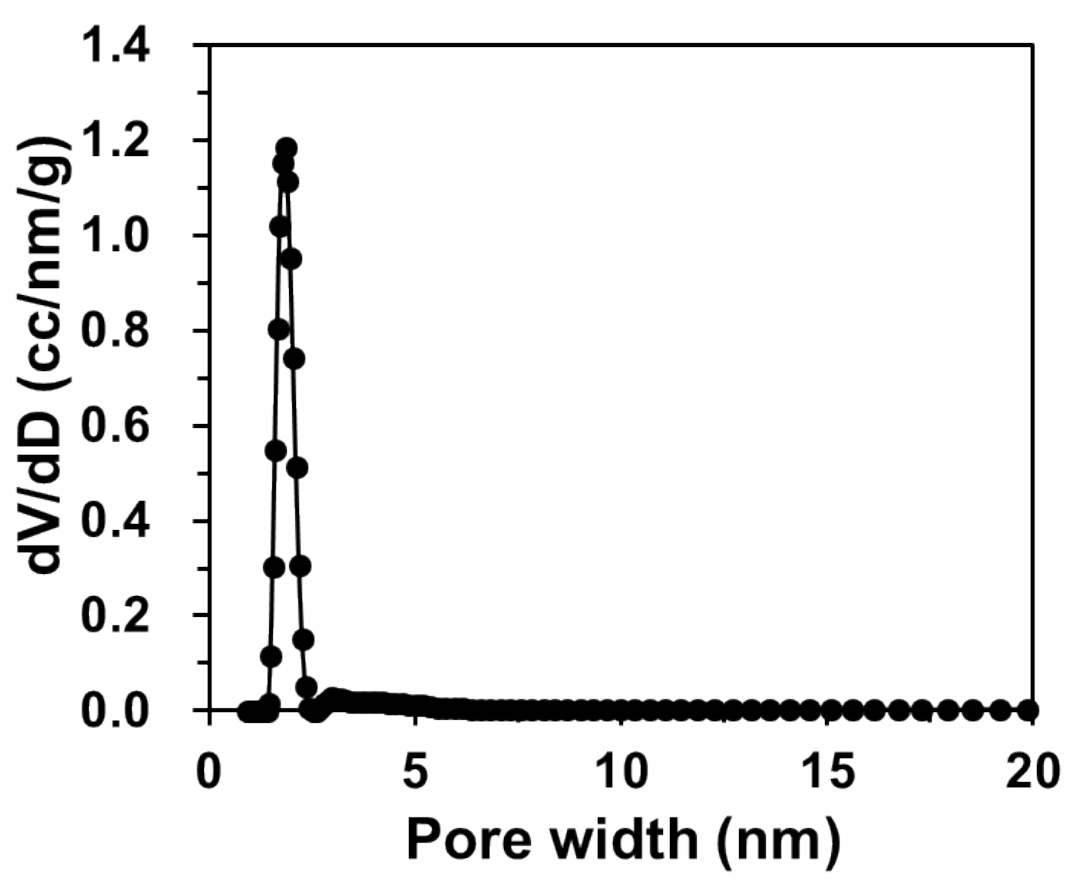

Supplementary Figure 49. Pore width distribution calculated by NLDFT model of TAPA-TFPA COF flower synthesized at $80^{\circ} \mathrm{C}$.
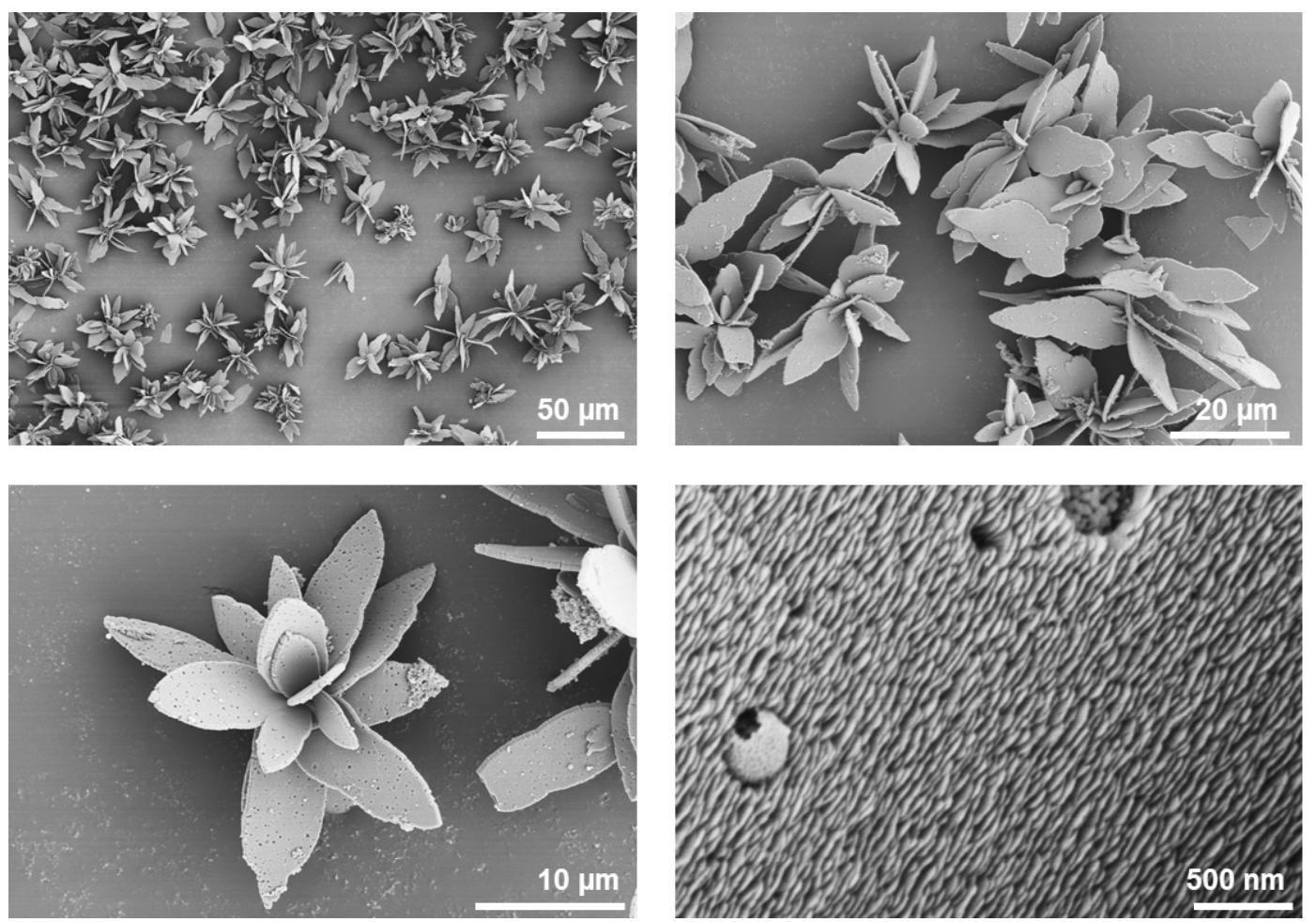

Supplementary Figure 50. FESEM images of the TAPA-TFPA COF flower after pyrolysis at $900{ }^{\circ} \mathrm{C}$. 


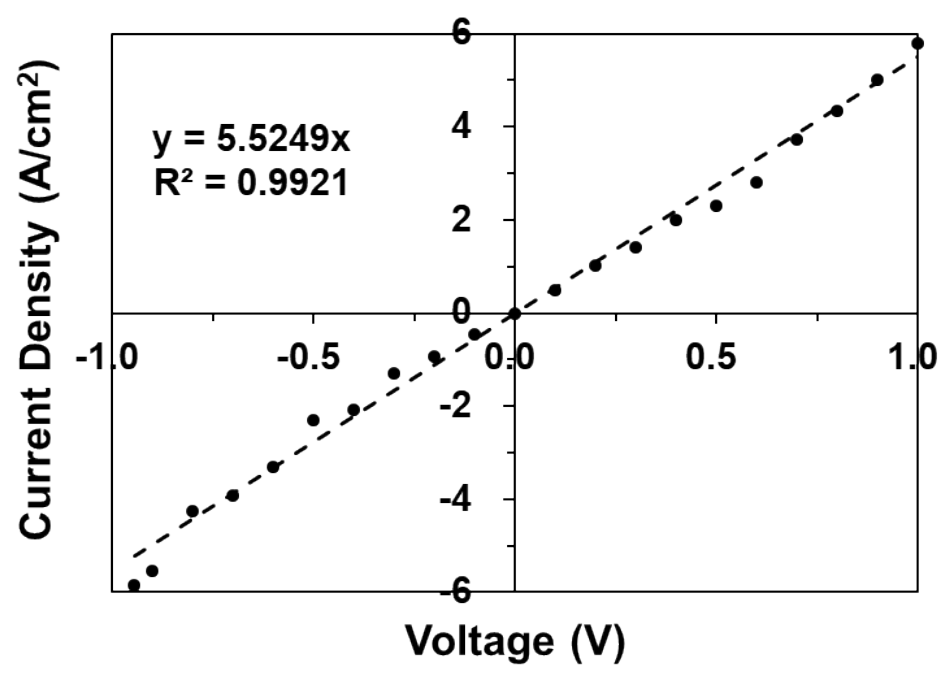

Supplementary Figure 51. Current Density varied with voltage. Conductivity is calculated as $384 \mathrm{~S} / \mathrm{m}$.
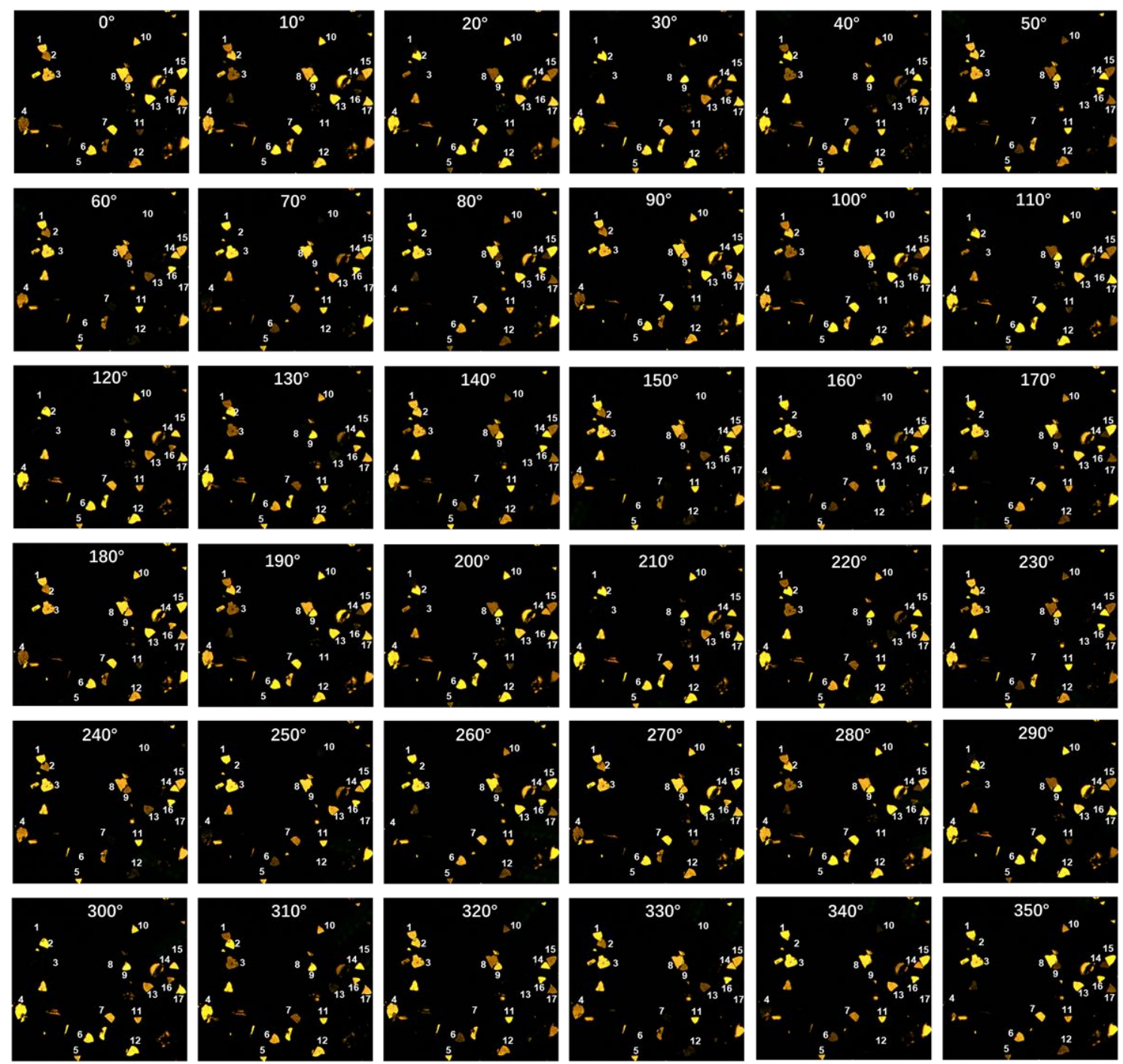

Supplementary Figure 52. Polarized light microscopy images at different rotation angle of the samples. 

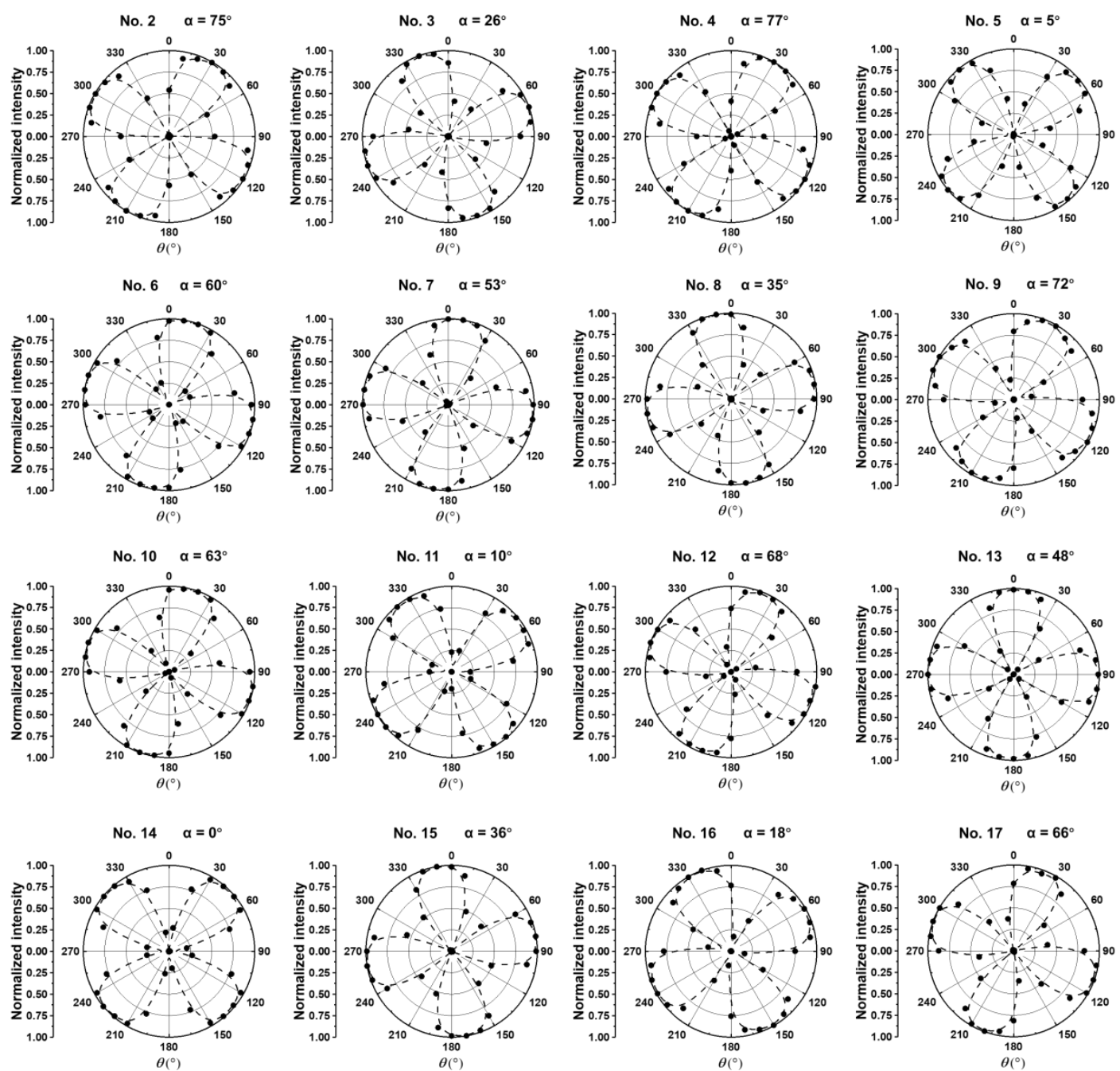

Supplementary Figure 53. Azimuthal plot of normalized brightness intensity response of the rotation angle of different COF petal from No.2 to 17 in Fig. 4B and Supplementary Figure 50. 

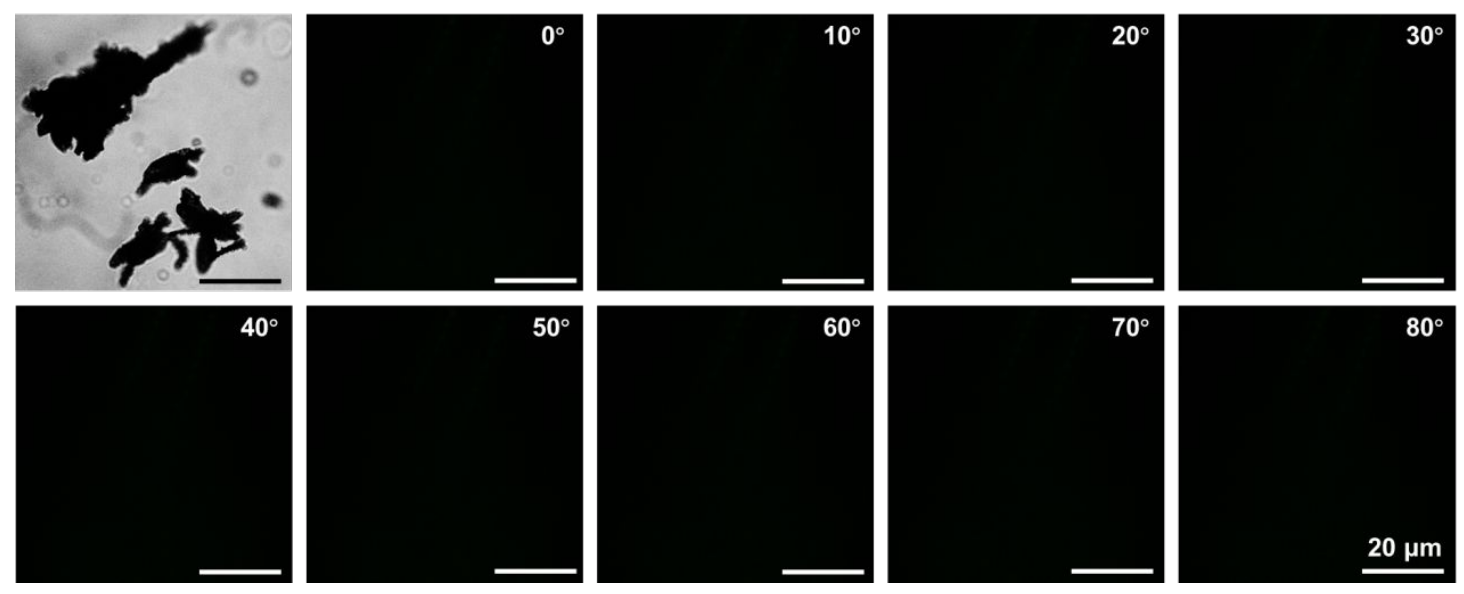

Supplementary Figure 54. Optical microscope (top left) and Polarized light microscopy images at different rotation angle of the TAPA-TFPA COF synthesized at $\mathrm{c}_{\mathrm{cat}}=0.1 \mathrm{mg} / \mathrm{ml}$ (SEM images see top left in Supplementary Figure 2.), other conditions are the same with COF flowers.
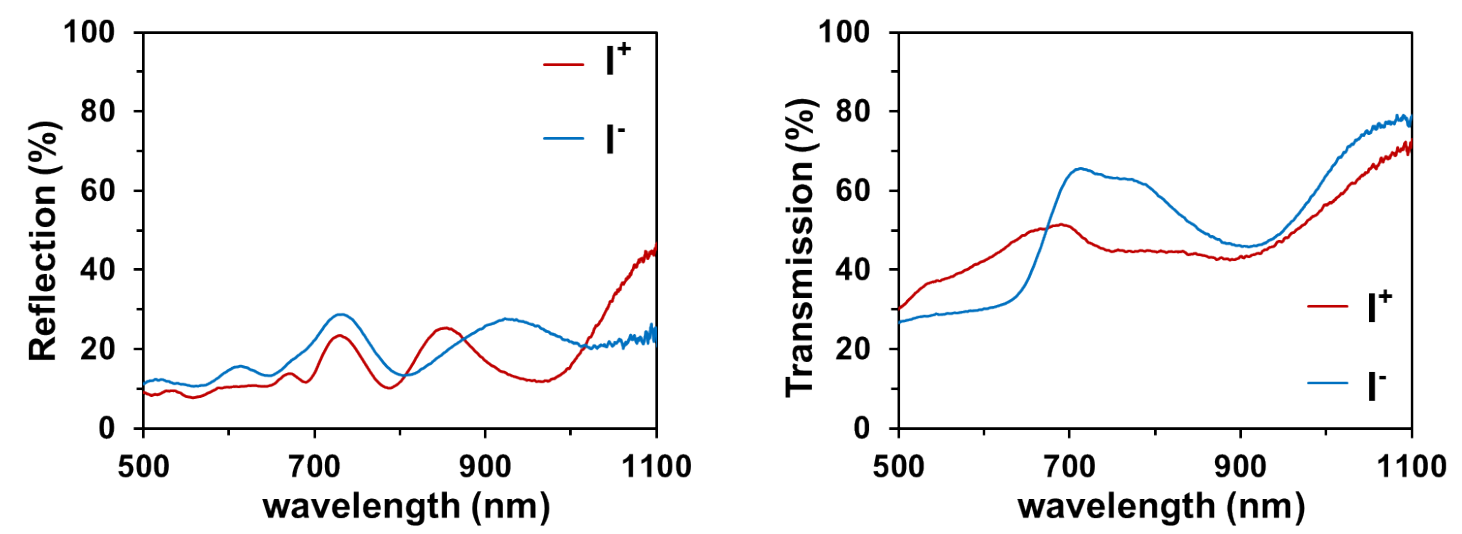

Supplementary Figure 55. Refection and Transmission spectra of polarized optical normal-incidence of two orthogonal linear polarized incident lights on a COF petal synthesized at $60^{\circ} \mathrm{C}$.
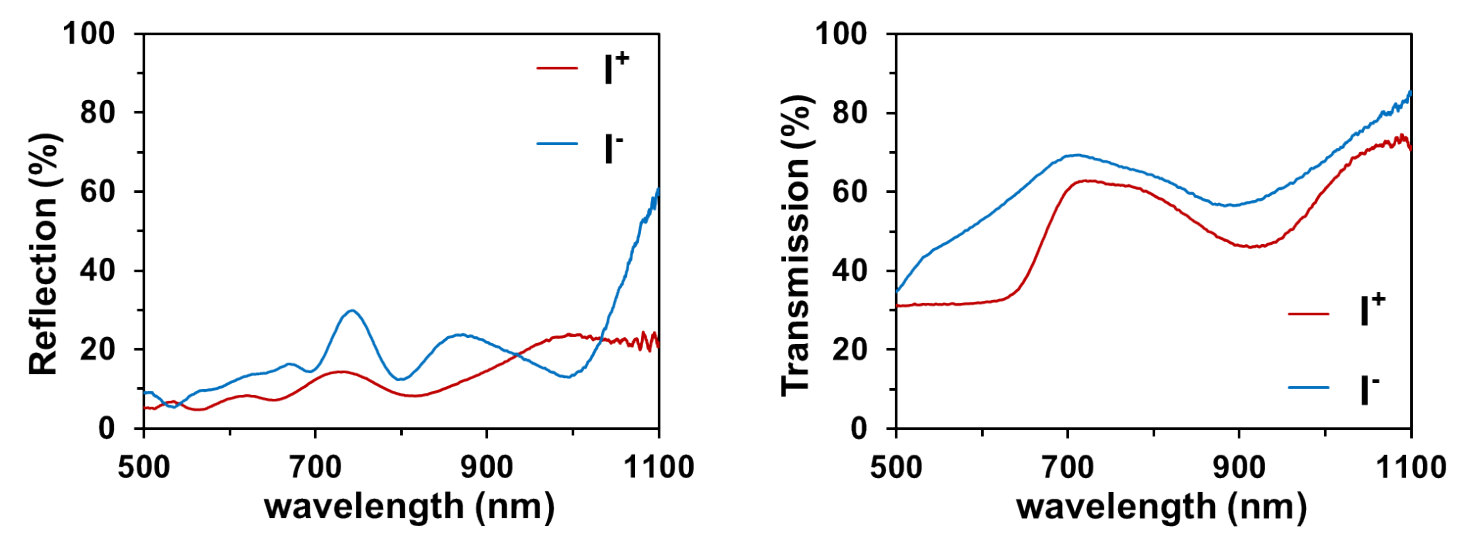

Supplementary Figure 56. Refection and Transmission spectra of polarized optical normal-incidence of two orthogonal linear polarized incident lights on a COF petal synthesized at $80^{\circ} \mathrm{C}$. 

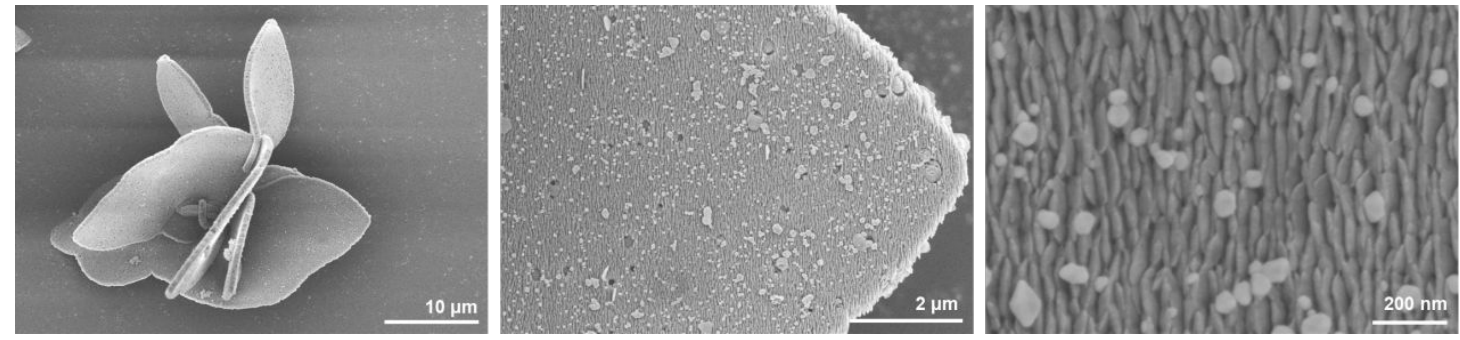

Supplementary Figure 57. FESEM images of COF flower with Ag nanoparticles.
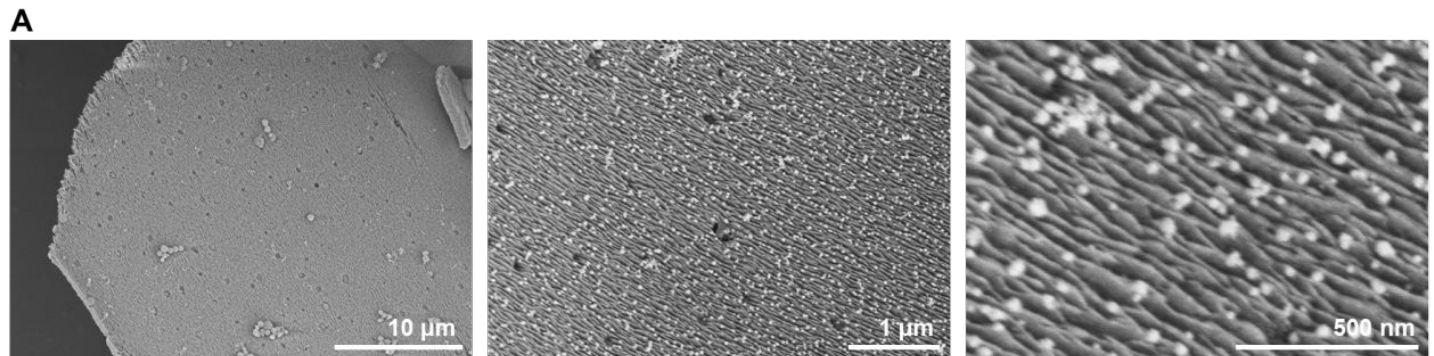

B
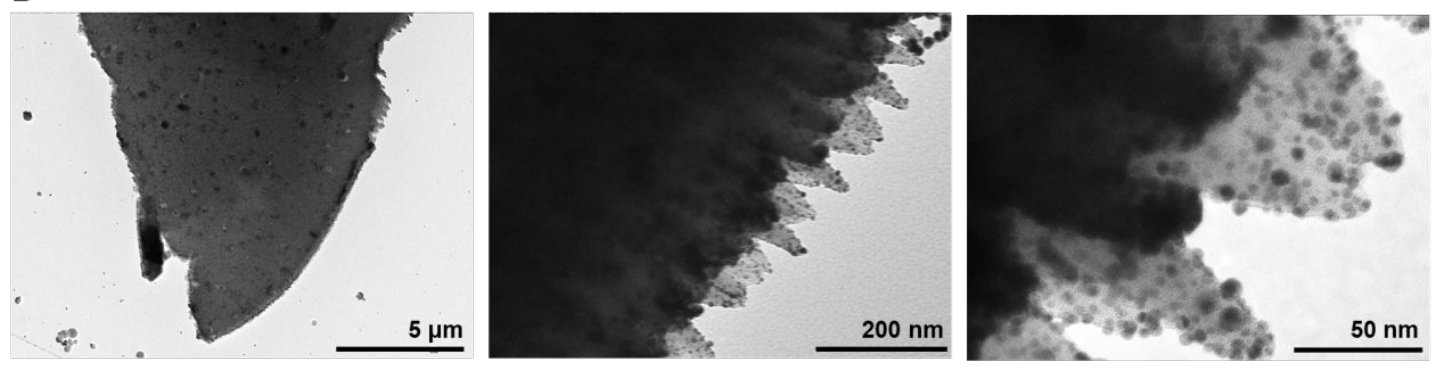

C
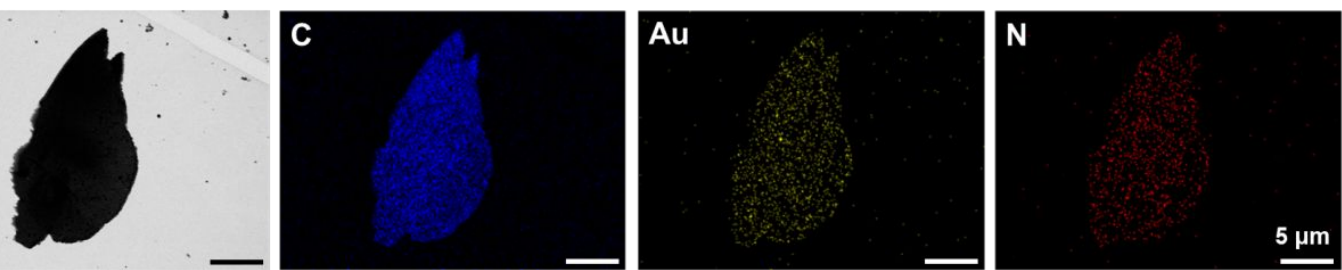

Supplementary Figure 58. FESEM (A), TEM (B) and elemental mapping of carbon, gold and nitrogen $(\mathrm{C})$ of COF flowers with $\mathrm{Au}$ nanoparticles. 

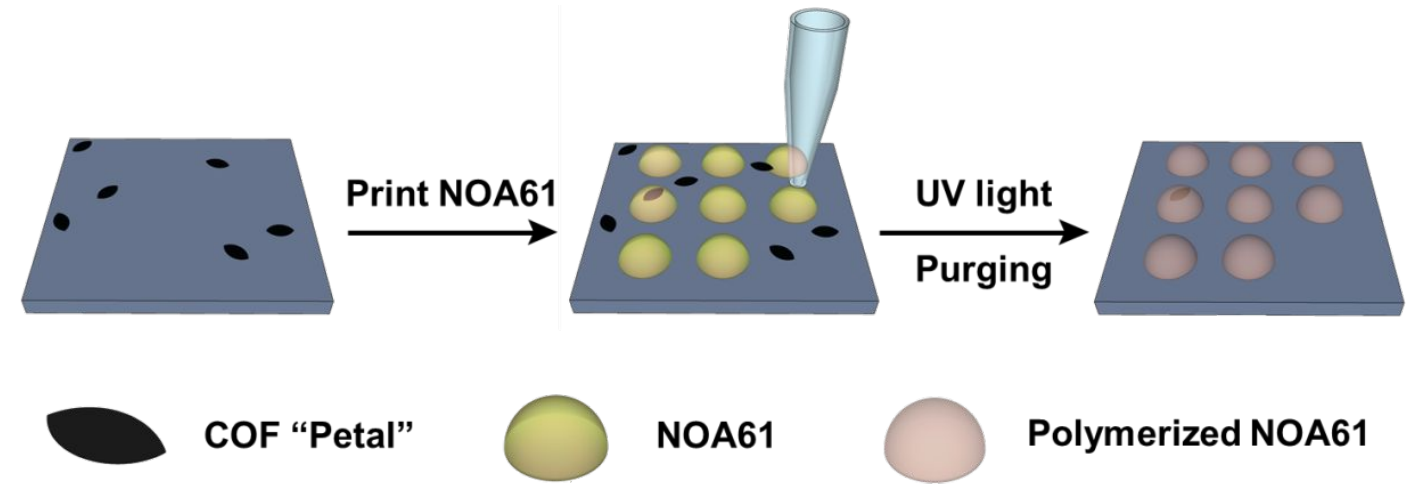

Supplementary Figure 59. Illustration of fabrication procedures for anticounterfeiting pattern.

$[2,1] 0^{\circ}, 20^{\circ}, 80^{\circ}$
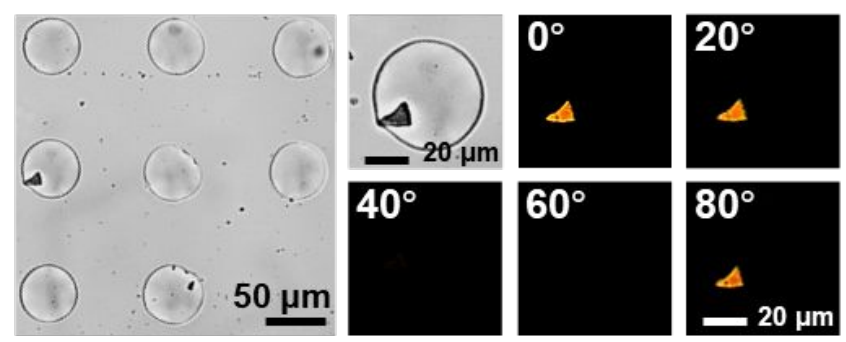

$80^{\circ}$

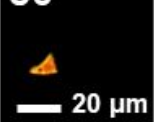

$[2,3] 0^{\circ}, 20^{\circ}, 40^{\circ}$,
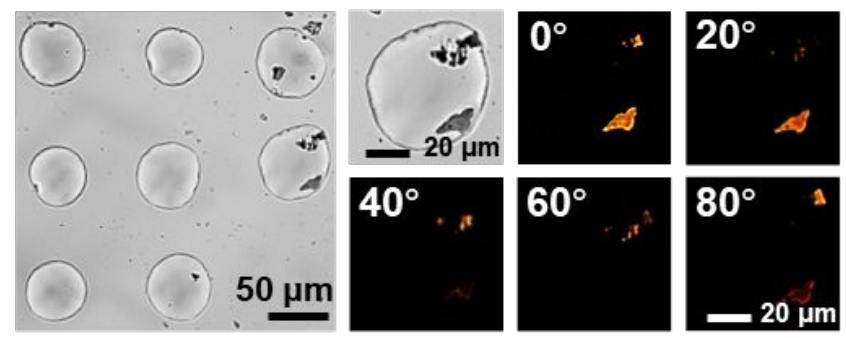

Supplementary Figure 60. Optical microscope and polarized optical microscopy images of two anti-counterfeiting pattern with digitized codes of $[2,1] 0^{\circ}, 20^{\circ}, 80^{\circ}$ and $[2,3] 0^{\circ}, 20^{\circ}, 40^{\circ}, 60^{\circ}, 80^{\circ}$, respectively. 


\section{A}

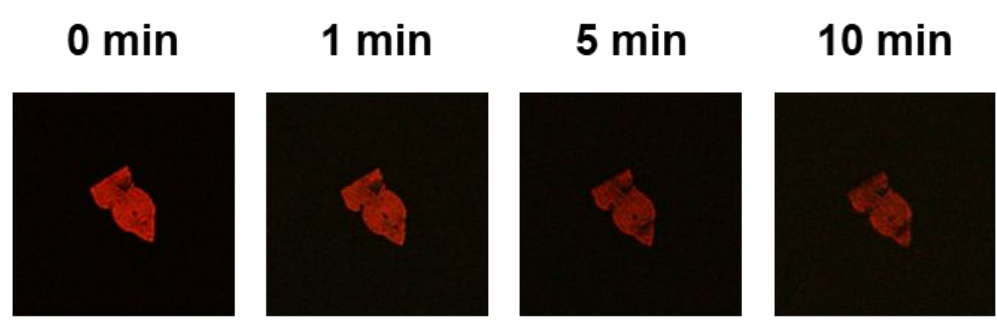

B

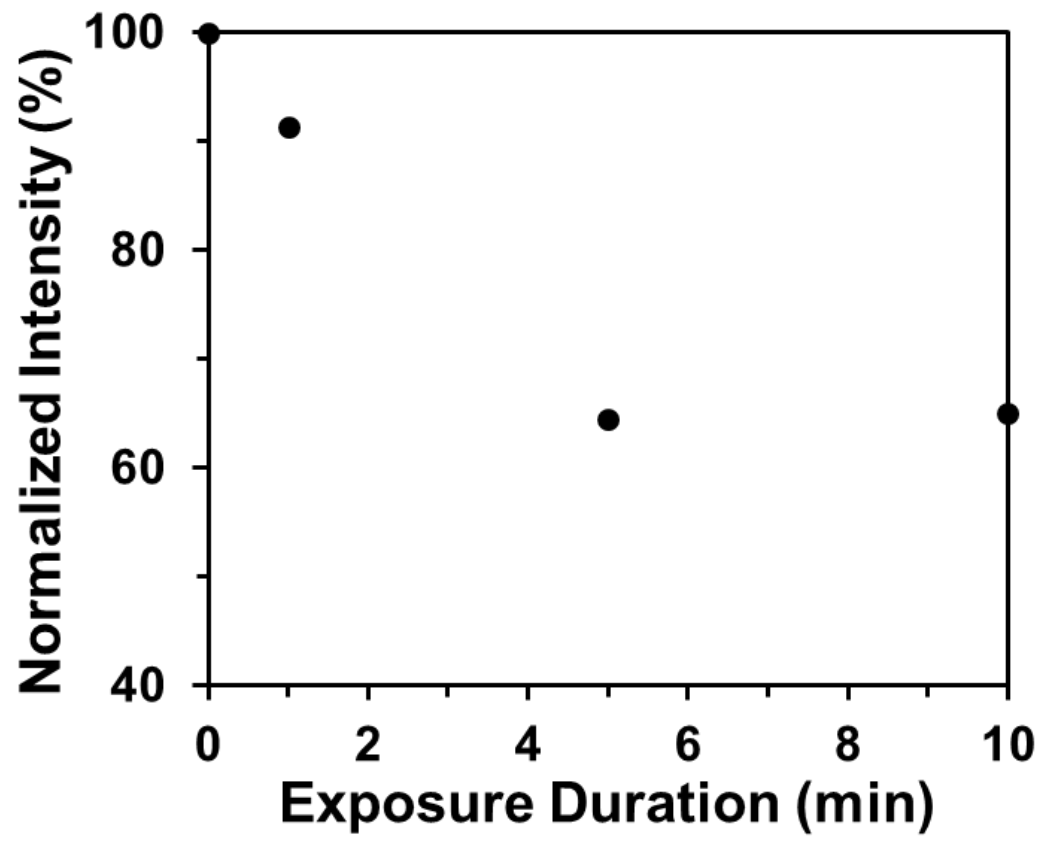

Supplementary Figure 61. Polarized optical microscopy images (A) and normalized intensity (B) of a petal sample after expose in iodine vapor for different time. 


\section{Reference:}

1 Peng, Y. et al. Ultrathin two-dimensional covalent organic framework nanosheets: preparation and application in highly sensitive and selective DNA detection. Journal of the American Chemical Society 139, 8698-8704 (2017).

2 El-Mahdy, A. F. et al. Strategic design of triphenylamine-and triphenyltriazine-based two-dimensional covalent organic frameworks for $\mathrm{CO} 2$ uptake and energy storage. Journal of Materials Chemistry A 6, 19532-19541 (2018).

3 Zhu, D. \& Verduzco, R. Ultralow Surface Tension Solvents Enable Facile COF Activation with Reduced Pore Collapse. (2020).

4 Feriante, C. H. et al. Rapid Synthesis of High Surface Area Imine-Linked 2D Covalent Organic Frameworks by Avoiding Pore Collapse During Isolation. Adv. Mater. 32, 1905776 (2020).

5 Sick, T. et al. Switching on and off interlayer correlations and porosity in 2D covalent organic frameworks. Journal of the American Chemical Society 141, 12570-12581 (2019). 